\title{
Infinitesimal CR automorphisms and stability groups of infinite-type models in $\mathbb{C}^{2}$
}

\author{
Atsushi Hayashimoto and Ninh Van Thu
}

\begin{abstract}
The purpose of this article is to give explicit descriptions for stability groups of real rigid hypersurfaces of infinite type in $\mathbb{C}^{2}$. The decompositions of infinitesimal $\mathrm{CR}$ automorphisms are also given.
\end{abstract}

\section{Introduction}

Let $M$ be a $\mathcal{C}^{\infty}$-smooth real hypersurface in $\mathbb{C}^{n}$, and let $p \in M$. We denote by $\operatorname{Aut}(M)$ the Cauchy-Riemann (CR) automorphism group of $M$, by $\operatorname{Aut}(M, p)$ the stability group of $M$, that is, those germs at $p$ of biholomorphisms mapping $M$ into itself and fixing $p$, and by $\mathfrak{a u t}(M, p)$ the set of germs of holomorphic vector fields in $\mathbb{C}^{n}$ at $p$ whose real part is tangent to $M$. We call this set the Lie algebra of infinitesimal CR automorphisms. We also denote by $\mathfrak{a u t}_{0}(M, p):=$ $\{H \in \mathfrak{a u t}(M, p): H(p)=0\}$.

For a real hypersurface in $\mathbb{C}^{n}$, the stability group and the Lie algebra of infinitesimal CR automorphisms are not easy to describe explicitly; besides, they are unknown in most cases. But, the study of $\operatorname{Aut}(M, p)$ and $\mathfrak{a u t}(M, p)$ of special types of hypersurfaces is given in [CM], [EKS1], [EKS2], [K1], [K2], [K3], [KM], [KMZ], [S2], and [S1]. For instance, explicit forms of the stability groups of models (see detailed definition in [K1], [KMZ]) have been obtained in [EKS2], [K1], [K2], and [KMZ]. However, these results are known for Levi nondegenerate hypersurfaces or, more generally, for Levi degenerate hypersurfaces of finite type in the sense of D'Angelo [D].

In this article, we give explicit descriptions for the Lie algebra of infinitesimal $\mathrm{CR}$ automorphisms and for the stability group of an infinite-type model $\left(M_{P}, 0\right)$ in $\mathbb{C}^{2}$ which is defined by

$$
M_{P}:=\left\{\left(z_{1}, z_{2}\right) \in \mathbb{C}^{2}: \operatorname{Re} z_{1}+P\left(z_{2}\right)=0\right\},
$$

Kyoto Journal of Mathematics, Vol. 56, No. 2 (2016), 441-464

DOI $10.1215 / 21562261-3478925$, (C) 2016 by Kyoto University

Received October 20, 2014. Revised March 30, 2015. Accepted April 15, 2015.

2010 Mathematics Subject Classification: Primary 32M05; Secondary 32H02, 32H50, 32T25.

Thu's work was supported in part by a National Research Foundation grant 2011-0030044 (Science

Research Center-The Center for Geometry and its Applications (SRC-GAIA)) of the Ministry of

Education, Republic of Korea. 
where $P$ is a nonzero germ of a real-valued $\mathcal{C}^{\infty}$-smooth function at 0 vanishing to infinite order at $z_{2}=0$.

To state these results more precisely, we establish some notation. Denote by $\mathrm{G}_{2}\left(M_{P}, 0\right)$ the set of all $\mathrm{CR}$ automorphisms of $M_{P}$ defined by

$$
\left(z_{1}, z_{2}\right) \mapsto\left(z_{1}, g_{2}\left(z_{2}\right)\right)
$$

for some holomorphic function $g_{2}$ with $g_{2}(0)=0$ and $\left|g_{2}{ }^{\prime}(0)\right|=1$ defined on a neighborhood of the origin in $\mathbb{C}$ satisfying that $P\left(g_{2}\left(z_{2}\right)\right) \equiv P\left(z_{2}\right)$. Also denote by $\Delta_{\epsilon_{0}}$ a disk with center at the origin and radius $\epsilon_{0}$, and denote by $\Delta_{\epsilon_{0}}^{*}$ a punctured disk $\Delta_{\epsilon_{0}} \backslash\{0\}$.

Let $P: \Delta_{\epsilon_{0}} \rightarrow \mathbb{R}$ be a $\mathcal{C}^{\infty}$-smooth function. Let us denote by $S_{\infty}(P)=\{z \in$ $\left.\Delta_{\epsilon_{0}}: \nu_{z}(P)=+\infty\right\}$, where $\nu_{z}(P)$ is the vanishing order of $P(z+\zeta)-P(z)$ at $\zeta=0$, and denote by $P_{\infty}\left(M_{P}\right)$ the set of all points of infinite type in $M_{P}$.

\section{REMARK 1}

It is not hard to see that $P_{\infty}\left(M_{P}\right)=\left\{\left(i t-P\left(z_{2}\right), z_{2}\right): t \in \mathbb{R}, z_{2} \in S_{\infty}(P)\right\}$.

\section{REMARK 2}

In the case that $P \not \equiv 0, \mathrm{G}_{2}\left(M_{P}, 0\right)$ contains only $\mathrm{CR}$ automorphisms of $M_{P}$ defined by

$$
\left(z_{1}, z_{2}\right) \mapsto\left(z_{1}, g_{2}\left(z_{2}\right)\right)
$$

where $g_{2}$ is a conformal map with $g_{2}(0)=0$ satisfying $P\left(g_{2}\left(z_{2}\right)\right) \equiv P\left(z_{2}\right)$ and either $g_{2}{ }^{\prime}(0)=e^{2 \pi i p / q}(p, q \in \mathbb{Z})$ and $g_{2}{ }^{q}=$ id or $g_{2}{ }^{\prime}(0)=e^{2 \pi i \theta}$ for some $\theta \in \mathbb{R} \backslash \mathbb{Q}$ (see Lemma 3 in Section 2 and Lemmas 5 and 6 in Section 3).

The first aim of this article is to prove the following two theorems, which give a decomposition of the infinitesimal CR automorphisms and an explicit description for stability groups of infinite-type models. In what follows, all functions, mappings, hypersurfaces, and so on are understood to be germs at the reference points, and we will not refer to them if there is no confusion.

\section{THEOREM 1}

Let $\left(M_{P}, 0\right)$ be a real $\mathcal{C}^{\infty}$-smooth hypersurface defined by the equation $\rho(z):=$ $\rho\left(z_{1}, z_{2}\right)=\operatorname{Re} z_{1}+P\left(z_{2}\right)=0$, where $P$ is a $\mathcal{C}^{\infty}$-smooth function on a neighborhood of the origin in $\mathbb{C}$ satisfying the conditions:

(i) $P\left(z_{2}\right) \not \equiv 0$ on a neighborhood of $z_{2}=0$, and

(ii) the connected component of 0 in $S_{\infty}(P)$ is $\{0\}$.

Then the following assertions hold.

(a) The Lie algebra $\mathfrak{g}=\mathfrak{a} \mathfrak{u}\left(M_{P}, 0\right)$ admits the decomposition

$$
\mathfrak{g}=\mathfrak{g}_{-1} \oplus \mathfrak{a u t}_{0}\left(M_{P}, 0\right),
$$

where $\mathfrak{g}_{-1}=\left\{i \beta \partial_{z_{1}}: \beta \in \mathbb{R}\right\}$. 
(b) If $\mathfrak{a} \mathfrak{u t} \mathfrak{t}_{0}\left(M_{P}, 0\right)$ is trivial, then

$$
\operatorname{Aut}\left(M_{P}, 0\right)=\mathrm{G}_{2}\left(M_{P}, 0\right) .
$$

\section{REMARK 3}

The condition (ii) simply tells us that $M_{P}$ is of infinite type. Moreover, the connected component of 0 in $P_{\infty}\left(M_{P}\right)$ is the set $\{(i t, 0): t \in \mathbb{R}\}$, which plays a key role in the proof of this theorem.

In the case that the connected component of 0 in $S_{\infty}(P)$ is not $\{0\}$, such as when $M_{P}$ is tubular, we have the following theorem.

\section{THEOREM 2}

Let $\tilde{P}$ be a $\mathcal{C}^{\infty}$-smooth function defined on a neighborhood of 0 in $\mathbb{R}$ satisfying

(i) $\tilde{P}(x) \not \equiv 0$ on a neighborhood of $x=0$ in $\mathbb{R}$, and

(ii) the connected component of 0 in $S_{\infty}(\tilde{P})$ is $\{0\}$.

Denote by $P$ a function defined by setting $P\left(z_{2}\right):=\tilde{P}\left(\operatorname{Re} z_{2}\right)$. Then the following assertions hold.

(a) $\mathfrak{a u t}_{0}\left(M_{P}, 0\right)=0$ and the Lie algebra $\mathfrak{g}=\mathfrak{a} \mathfrak{u}\left(M_{P}, 0\right)$ admits the decomposition

$$
\mathfrak{g}=\mathfrak{g}_{-1} \oplus \mathfrak{g}_{0},
$$

where $\mathfrak{g}_{-1}=\left\{i \beta \partial_{z_{1}}: \beta \in \mathbb{R}\right\}$ and $\mathfrak{g}_{0}=\left\{i \beta \partial_{z_{2}}: \beta \in \mathbb{R}\right\}$.

(b) $\operatorname{Aut}\left(M_{P}, 0\right)=\{i d\}$.

(c) If $S_{\infty}(\tilde{P})=\{0\}$, then $\operatorname{Aut}\left(M_{P}\right)=\mathrm{T}^{1}\left(M_{P}\right) \oplus \mathrm{T}^{2}\left(M_{P}\right)=\left\{\left(z_{1}, z_{2}\right) \mapsto\left(z_{1}+\right.\right.$ $\left.\left.i t, z_{2}+i s\right): t, s \in \mathbb{R}\right\}$, where $\mathrm{T}^{1}\left(M_{P}\right)=\left\{\left(z_{1}, z_{2}\right) \mapsto\left(z_{1}+i t, z_{2}\right): t \in \mathbb{R}\right\}$ and $\mathrm{T}^{2}\left(M_{P}\right)=\left\{\left(z_{1}, z_{2}\right) \mapsto\left(z_{1}, z_{2}+i t\right): t \in \mathbb{R}\right\}$.

These theorems show that the special conditions of defining functions determine the forms of holomorphic vector fields. Conversely, the second aim of this article is to show that holomorphic vector fields determine the form of defining functions. This is, in some sense, the converse of Example 2 in Section 6, which holds generally. Namely, we prove the following.

\section{THEOREM 3}

Let $\left(M_{P}, 0\right)$ be a $\mathcal{C}^{\infty}$-smooth hypersurface defined by the equation $\rho(z):=$ $\rho\left(z_{1}, z_{2}\right)=\operatorname{Re} z_{1}+P\left(z_{2}\right)=0$, satisfying the conditions:

(i) the connected component of $z_{2}=0$ in the zero set of $P$ is $\{0\}$, and

(ii) $P$ vanishes to infinite order at $z_{2}=0$.

Then any holomorphic vector field vanishing at the origin tangent to $\left(M_{P}, 0\right)$ is either identically zero or, after a change of variable in $z_{2}$, of the form $i \beta z_{2} \partial_{z_{2}}$ 
for some nonzero real number $\beta$, in which case $M_{P}$ is rotationally symmetric; that is, $P\left(z_{2}\right)=P\left(\left|z_{2}\right|\right)$.

The organization of this article is as follows. In Section 2, we prove three lemmas which we use in the proof of theorems. In Section 3, we give a description of stability groups, and proofs of Theorems 1 and 2 are given in Section 4. In Section 5, we prove Theorem 3 and the lemmas needed to prove it. In Section 6, we introduce some examples. Finally, two theorems are presented in the Appendix.

\section{Preliminaries}

In this section, we shall recall some definitions and introduce three lemmas which are used to prove Theorems 1 and 2 .

\section{DEFINITION 1}

Let $g_{1}, g_{2}$ be two conformal maps with $g_{1}(0)=g_{2}(0)=0$. We say that $g_{1}$ and $g_{2}$ are holomorphically locally conjugated if there exists a biholomorphism $\varphi$ with $\varphi(0)=0$ such that

$$
g_{1} \equiv \varphi^{-1} \circ g_{2} \circ \varphi
$$

\section{DEFINITION 2}

Let $g$ be a conformal map with $g(0)=0$.

(i) If $g^{\prime}(0)=1$, then we say that $g$ is tangent to the identity.

(ii) If $g^{\prime}(0)=e^{2 \pi i p / q}, p, q \in \mathbb{Z}$, then we say that $g$ is parabolic.

(iii) If $g^{\prime}(0)=e^{2 \pi i \theta}$ for some $\theta \in \mathbb{R} \backslash \mathbb{Q}$, then we say that $g$ is elliptic.

The following lemma is a slight generalization of [N1, Lemma 2].

\section{LEMMA 1}

Let $P$ be a $\mathcal{C}^{\infty}$-smooth function on $\Delta_{\epsilon_{0}}\left(\epsilon_{0}>0\right)$ satisfying $\nu_{0}(P)=+\infty$ and $P(z) \not \equiv 0$. Suppose that there exists a conformal map $g$ on $\Delta_{\epsilon_{0}}$ with $g(0)=0$ such that

$$
P(g(z))=(\beta+o(1)) P(z), \quad z \in \Delta_{\epsilon_{0}},
$$

for some $\beta \in \mathbb{R}^{*}$. Then $\left|g^{\prime}(0)\right|=1$.

Proof

Suppose that there exist a conformal map $g$ with $g(0)=0$ and a $\beta \in \mathbb{R}^{*}$ such that $P(g(z))=(\beta+o(1)) P(z)$ holds for $z \in \Delta_{\epsilon_{0}}$. Then, we have

$$
P(g(z))=(\beta+\gamma(z)) P(z), \quad z \in \Delta_{\epsilon_{0}},
$$

where $\gamma$ is a function defined on $\Delta_{\epsilon_{0}}$ with $\gamma(z) \rightarrow 0$ as $z \rightarrow 0$, which implies that there exists $\delta_{0}>0$ such that $|\gamma(z)|<|\beta| / 2$ for any $z \in \Delta_{\delta_{0}}$. We consider the following cases. 
Case 1: $0<\left|g^{\prime}(0)\right|<1$. In this case, we can choose $\delta_{0}$ and $\alpha$ with $0<\delta_{0}<\epsilon_{0}$ and $\left|g^{\prime}(0)\right|<\alpha<1$ such that $|g(z)| \leq \alpha|z|$ for all $z$ in $\Delta_{\delta_{0}}$. Fix a point $z_{0} \in \Delta_{\delta_{0}}^{*}$ with $P\left(z_{0}\right) \neq 0$. Then, for each positive integer $n$, we get

$$
\begin{aligned}
\left|P\left(g^{n}\left(z_{0}\right)\right)\right| & =\left|\left(\beta+\gamma\left(g^{n-1}\left(z_{0}\right)\right)\right)\right|\left|P\left(g^{n-1}\left(z_{0}\right)\right)\right|=\cdots \\
& =\left|\left(\beta+\gamma\left(g^{n-1}\left(z_{0}\right)\right)\right)\right| \cdots\left|\left(\beta+\gamma\left(z_{0}\right)\right)\right|\left|P\left(z_{0}\right)\right| \\
& \geq\left(|\beta|-\left|\gamma\left(g^{n-1}\left(z_{0}\right)\right)\right|\right) \cdots\left(|\beta|-\left|\gamma\left(z_{0}\right)\right|\right)\left|P\left(z_{0}\right)\right| \\
& \geq(|\beta| / 2)^{n}\left|P\left(z_{0}\right)\right|,
\end{aligned}
$$

where $g^{n}$ denotes the composition of $g$ with itself $n$ times. Moreover, since $0<$ $\alpha<1$, there exists a positive integer $m_{0}$ such that $\left|\alpha^{m_{0}}\right|<|\beta| / 2$. Notice that $0<\left|g^{n}\left(z_{0}\right)\right| \leq \alpha^{n}\left|z_{0}\right|$ for any $n \in \mathbb{N}$. Then it follows from (1) that

$$
\frac{\left|P\left(g^{n}\left(z_{0}\right)\right)\right|}{\left|g^{n}\left(z_{0}\right)\right|^{m_{0}}} \geq \frac{\left|P\left(z_{0}\right)\right|}{\left|z_{0}\right|^{m_{0}}}\left(\frac{|\beta| / 2}{\alpha^{m_{0}}}\right)^{n} .
$$

This yields that $\left|P\left(g^{n}\left(z_{0}\right)\right)\right| /\left|g^{n}\left(z_{0}\right)\right|^{m_{0}} \rightarrow+\infty$ as $n \rightarrow \infty$, which contradicts the fact that $P$ vanishes to infinite order at 0 .

Case 2: $1<\left|g^{\prime}(0)\right|$. Since $P(g(z))=(\beta+o(1)) P(z)$ for all $z \in \Delta_{\epsilon_{0}}$, it follows that $P\left(g^{-1}(z)\right)=(1 / \beta+o(1)) P(z)$ for all $z \in \Delta_{\epsilon_{0}}$, which is impossible because of Case 1 .

Altogether, $\left|g^{\prime}(0)\right|=1$, and the proof is thus complete.

\section{LEMMA 2}

Let $f:[-r, r] \rightarrow \mathbb{R}(r>0)$ be a continuous function satisfying $f(0)=0$ and $f \not \equiv 0$. If $\beta$ is a real number such that

$$
f(t+\beta f(t))=f(t)
$$

for every $t \in[-r, r]$ with $t+\beta f(t) \in[-r, r]$, then $\beta=0$.

\section{Proof}

Suppose, to derive a contradiction, that there exists a $\beta \neq 0$ such that $f(t+$ $\beta f(t))=f(t)$ for every $t \in[-r, r]$ with $t+\beta f(t) \in[-r, r]$. Then we have

$$
\begin{aligned}
f(t) & =f(t+\beta f(t))=f(t+\beta f(t)+\beta f(t+\beta f(t))) \\
& =f(t+2 \beta f(t))=\cdots=f(t+m \beta f(t))
\end{aligned}
$$

for every $m \in \mathbb{N}$ and for every $t \in[-r, r]$ with $t+m \beta f(t) \in[-r, r]$.

Let $t_{0} \in[-r, r]$ be such that $f\left(t_{0}\right) \neq 0$. Then since $f$ is uniformly continuous on $[-r, r]$, for every $\epsilon>0$ there exists $\delta>0$ such that, for every $t_{1}, t_{2} \in[-r, r]$ with $\left|t_{1}-t_{2}\right|<\delta$, we have that $\left|f\left(t_{1}\right)-f\left(t_{2}\right)\right|<\epsilon / 2$. On the other hand, since $f(t) \rightarrow 0$ as $t \rightarrow 0$ and since $f \not \equiv 0$, one can find $t \in[-\delta / 2, \delta / 2]$ such that $|\beta f(t)|<\delta$ and $0<|f(t)|<\epsilon / 2$. Therefore, there exists an integer $m$ such that $\left|t+m \beta f(t)-t_{0}\right|<$ 
$\delta$, and thus by (2) one has

$$
\begin{aligned}
\left|f\left(t_{0}\right)\right| & \leq\left|f(t+m \beta f(t))-f\left(t_{0}\right)\right|+|f(t+m \beta f(t))| \\
& <\epsilon / 2+|f(t)|<\epsilon / 2+\epsilon / 2=\epsilon .
\end{aligned}
$$

This implies that $f\left(t_{0}\right)=0$, which is a contradiction. Hence, the proof is complete.

\section{LEMMA 3}

Let $P$ be a nonzero $\mathcal{C}^{\infty}$-smooth function with $P(0)=0$, and let $g$ be a conformal map satisfying $g(0)=0,\left|g^{\prime}(0)\right|=1$, and $g \neq \mathrm{id}$. If there exists a real number $\delta \in \mathbb{R}^{*}$ such that $P(g(z)) \equiv \delta P(z)$, then $\delta=1$. Moreover, we have either $g^{\prime}(0)=$ $e^{2 \pi i p / q}(p, q \in \mathbb{Z})$ and $g^{q}=$ id or $g^{\prime}(0)=e^{2 \pi i \theta}$ for some $\theta \in \mathbb{R} \backslash \mathbb{Q}$.

\section{Proof}

Replacing $g$ by its inverse if necessary, one can assume that $|\delta| \geq 1$. Now we divide the proof into three cases as follows.

Case 1: $g^{\prime}(0)=1$. As a consequence of the Leau-Fatou flower theorem (see Theorem 4 in Appendix A.1), there exists a point $z$ in a small neighborhood of the origin with $P(z) \neq 0$ such that $g^{n}(z) \rightarrow 0$ as $n \rightarrow \infty$. Since $P\left(g^{n}(z)\right)=(\delta)^{n} P(z)$ and $\lim _{n \rightarrow+\infty} P\left(g^{n}(z)\right)=P(0)=0$, we have $0<|\delta|<1$, which is a contradiction.

Case 2: $\lambda:=g^{\prime}(0)=e^{2 \pi i p / q}(p, q \in \mathbb{Z})$. Suppose that $g^{q}=\mathrm{id}$; then by [A, Proposition 3.2], there exists $z$ in a small neighborhood of 0 satisfying $P(z) \neq 0$ such that the orbit $\left\{g^{n}(z)\right\}$ is contained in a relativity compact subset of some punctured neighborhood. Therefore, by the assumption that $P(g(z)) \equiv \delta P(z)$, the sequence $\left\{\delta^{n}\right\}$ must be convergent. This means that $\delta=1$. In the case of $g^{q} \neq \mathrm{id}$, we have $g^{q}(z)=z+\cdots$ and $P\left(g^{q}(z)\right) \equiv \delta^{q} P(z)$. This is absurd because of Case 1 with $g$ being replaced by $g^{q}$.

Case 3: $\lambda:=g^{\prime}(0)=e^{2 \pi i \theta}(\theta \notin \mathbb{Q})$. By [A, Proposition 4.4], we may assume that there exists $z$ in a small neighborhood of 0 satisfying $P(z) \neq 0$ such that the orbit $\left\{g^{n}(z)\right\}$ is contained in a relativity compact subset of some punctured neighborhood. Therefore, the same argument as in Case 2 shows that $\delta=1$. Altogether, the proof is complete.

\section{Explicit description for $\mathrm{G}_{2}\left(M_{P}, 0\right)$}

In this section, we are going to give an explicit description for the subgroup $\mathrm{G}_{2}\left(M_{P}, 0\right)$ of the stability group of $M_{P}$. By virtue of Lemma $3, \mathrm{G}_{2}\left(M_{P}, 0\right)$ contains only $\mathrm{CR}$ automorphisms of $M_{P}$ defined by

$$
\left(z_{1}, z_{2}\right) \mapsto\left(z_{1}, g_{2}\left(z_{2}\right)\right)
$$

where $g_{2}$ is either parabolic or elliptic. Conversely, given either a parabolic $g$ with $g^{q}=$ id for some positive integer $q$ or an elliptic $g$, we shall show that there exist some infinite-type models $\left(M_{P}, 0\right)$ such that the mapping $\left(z_{1}, z_{2}\right) \mapsto\left(z_{1}, g\left(z_{2}\right)\right)$ belongs to $\mathrm{G}_{2}\left(M_{P}, 0\right)$.

First of all, we need the following lemma. 


\section{LEMMA 4}

If $P\left(e^{2 \pi i \theta} z\right) \equiv P(z)$ for some $\theta \in \mathbb{R} \backslash \mathbb{Q}$, then $P(z) \equiv P(|z|)$; that is, $P$ is rotational.

Proof

We note that $P\left(e^{2 \pi n i \theta} z\right) \equiv P(z)$ for any $n \in \mathbb{N}$ and $\overline{\left\{e^{2 \pi n i \theta} z: n \in \mathbb{N}\right\}}=\mathbb{S}_{|z|}$, where $\mathbb{S}_{r}:=\{z \in \mathbb{C}:|z|=r\}$ for $r>0$. Therefore, because of the continuity of $P$, we conclude that $P(z) \equiv P(|z|)$.

\subsection{The parabolic case}

\section{LEMMA 5}

Let $g(z)=e^{2 \pi i p / q} z+\cdots$ be a conformal map, with $\lambda=e^{2 \pi i p / q}$ being a primitive root of unity. If $g^{q}=\mathrm{id}$, then there exists an infinite-type model $M_{P}$ such that $\left(z_{1}, z_{2}\right) \mapsto\left(z_{1}, g^{j}\left(z_{2}\right)\right)$ belongs to $\mathrm{G}_{2}\left(M_{P}, 0\right)$ for every $j=1,2, \ldots, q-1$.

\section{Proof}

Suppose that $g(z)=e^{2 \pi i p / q} z+\cdots$ is a conformal map such that $\lambda=e^{2 \pi i p / q}$ is a primitive root of unity satisfying $g^{q}=\mathrm{id}$. It is known that $g$ is holomorphically locally conjugated to $h(z)=\lambda z$ (see [A, Proposition 3.2]). Let $\tilde{P}$ be a $\mathcal{C}^{\infty}$-smooth function with $\nu_{0}(\tilde{P})=+\infty$. Define a $\mathcal{C}^{\infty}$-smooth function by setting

$$
P(z):=\tilde{P}(z)+\tilde{P}(g(z))+\cdots+\tilde{P}\left(g^{q-1}(z)\right) .
$$

Then it is easy to see that $P(g(z)) \equiv P(z)$. Thus, $f_{j}\left(z_{1}, z_{2}\right)=\left(z_{1}, g^{j}\left(z_{2}\right)\right) \in$ $\mathrm{G}_{2}\left(M_{P}, 0\right), j=1, \ldots, q-1$, are biholomorphic.

\section{REMARK 4}

In the case of $g^{q} \neq \mathrm{id}$, we have $g^{d}(z)=z+\cdots$, and therefore $P(z+\cdots)=$ $P\left(g^{q}(z)\right)=P(z)$. It follows from Lemma 3 that there is no infinite-type model $M_{P}$ satisfying $P \not \equiv 0$ on some petal such that $\left(z_{1}, z_{2}\right) \mapsto\left(z_{1}, g\left(z_{2}\right)\right)$ belongs to $\mathrm{G}_{2}\left(M_{P}, 0\right)$.

\subsection{The elliptic cases}

\section{LEMMA 6}

Let $g(z)=e^{2 \pi i \theta} z+\cdots$ be a conformal map with $\theta \notin \mathbb{Q}$. Then there exists an infinite-type formal model $M_{P}$ such that $\left(z_{1}, z_{2}\right) \mapsto\left(z_{1}, g\left(z_{2}\right)\right)$ belongs to $\mathrm{G}_{2}\left(M_{P}, 0\right)$. Moreover, $M_{P}$ is biholomorphically equivalent to a rotationally symmetric model $M_{\tilde{P}}$.

Proof

Suppose that $g(z)=e^{2 \pi i \theta} z+\cdots$ is a conformal map with $\theta \notin \mathbb{Q}$. Then it is known that $g$ is formally locally conjugated to $R_{\theta}(z)=e^{2 \pi i \theta} z$ (see [A, Proposition 4.4]), that is, there exists a formally conformal map $\varphi$ at 0 with $\varphi(0)=0$ such that

$$
g=\varphi^{-1} \circ R_{\theta} \circ \varphi
$$


Let $\tilde{P}$ be a rotational $\mathcal{C}^{\infty}$-smooth function with $\nu_{0}(\tilde{P})=+\infty$. Define a $\mathcal{C}^{\infty}$ smooth formal function by setting

$$
P(z)=\tilde{P}(\varphi(z))=\tilde{P}(|\varphi(z)|) .
$$

Then $P(g(z))=\tilde{P}(\varphi \circ g(z))=\tilde{P}\left(R_{\theta} \circ \varphi(z)\right)=\tilde{P}\left(\left|R_{\theta} \circ \varphi(z)\right|\right)=\tilde{P}(|\varphi(z)|)=P(z)$. This means that $\left(z_{1}, z_{2}\right) \mapsto\left(z_{1}, g\left(z_{2}\right)\right)$ belongs to $\mathrm{G}_{2}\left(M_{P}, 0\right)$. Moreover, $f_{t}\left(z_{1}, z_{2}\right):=\left(z_{1}, \varphi^{-1} \circ R_{t} \circ \varphi\left(z_{2}\right)\right)$ is a formal mapping in $\mathrm{G}_{2}\left(M_{P}, 0\right)$ for all $t \in \mathbb{R}$. In addition, it is easy to see that $M_{P}$ is biholomorphically equivalent to $M_{\tilde{P}}$, which is rotationally symmetric.

\section{Proofs of Theorems 1 and 2}

This section is devoted to the proofs of Theorems 1 and 2. For the sake of smooth exposition, we shall present these proofs in two sections.

\subsection{Proof of Theorem 1}

Proof of Theorem 1

(a) Let $H\left(z_{1}, z_{2}\right)=h_{1}\left(z_{1}, z_{2}\right) \partial_{z_{1}}+h_{2}\left(z_{1}, z_{2}\right) \partial_{z_{2}} \in \mathfrak{a u t}\left(M_{P}, 0\right)$ be arbitrary, and let $\left\{\phi_{t}\right\}_{t \in \mathbb{R}} \subset \operatorname{Aut}\left(M_{P}\right)$ be the one-parameter subgroup generated by $H$. Since $\phi_{t}$ is biholomorphic for every $t \in \mathbb{R}$, the set $\left\{\phi_{t}(0): t \in \mathbb{R}\right\}$ is contained in $P_{\infty}\left(M_{P}\right)$. We remark that the connected component of 0 in $P_{\infty}\left(M_{P}\right)$ is $\{(i s, 0): s \in \mathbb{R}\}$. Therefore, we have $\phi_{t}(0,0) \subset\{(i s, 0): s \in \mathbb{R}\}$. Consequently, we obtain $\operatorname{Re} h_{1}(0,0)=0$ and $h_{2}(0,0)=0$. Hence, the holomorphic vector field $H-i \beta \partial_{z_{1}}$, where $\beta:=$ $\operatorname{Im} h_{1}(0,0)$, belongs to $\mathfrak{a u t}_{0}\left(M_{P}, 0\right)$, which ends the proof.

(b) In the light of (a), we see that $\mathfrak{a} \mathfrak{u}\left(M_{P}, 0\right)=\mathfrak{g}_{-1}$, that is, it is generated by $i \partial_{z_{1}}$. Denote by $\left\{T_{t}\right\}_{t \in \mathbb{R}}$ the one-parameter subgroup generated by $i \partial_{z_{1}}$, that is, it is given by

$$
T_{t}\left(z_{1}, z_{2}\right)=\left(z_{1}+i t, z_{2}\right), \quad t \in \mathbb{R} .
$$

Let $f=\left(f_{1}, f_{2}\right) \in \operatorname{Aut}\left(M_{P}, 0\right)$ be arbitrary. We define the family of automorphisms $\left\{F_{t}\right\}_{t \in \mathbb{R}}$ by setting $F_{t}:=f \circ T_{-t} \circ f^{-1}$. Then it follows that $\left\{F_{t}\right\}_{t \in \mathbb{R}}$ is a one-parameter subgroup of $\operatorname{Aut}\left(M_{P}\right)$. Since $\mathfrak{a u t}\left(M_{P}, 0\right)=\mathfrak{g}_{-1}$, it follows that the holomorphic vector field generated by $\left\{F_{t}\right\}_{t \in \mathbb{R}}$ belongs to $\mathfrak{g}_{-1}$. This means that there exists a real number $\delta$ such that $F_{t}=T_{\delta t}$ for all $t \in \mathbb{R}$, which yields that

$$
f=T_{\delta t} \circ f \circ T_{t}, \quad t \in \mathbb{R} .
$$

We note that if $\delta=0$, then $f=f \circ T_{t}$ and thus $T_{t}=$ id for any $t \in \mathbb{R}$, which is a contradiction. Hence, we may assume that $\delta \neq 0$.

We shall prove that $\delta=-1$. Indeed, (3) is equivalent to

$$
\begin{aligned}
& f_{1}\left(z_{1}, z_{2}\right)=f_{1}\left(z_{1}+i t, z_{2}\right)+i \delta t, \\
& f_{2}\left(z_{1}, z_{2}\right)=f_{2}\left(z_{1}+i t, z_{2}\right)
\end{aligned}
$$

for all $t \in \mathbb{R}$. This implies that $\frac{\partial}{\partial z_{1}} f_{1}\left(z_{1}, z_{2}\right)=-\delta$ and $\frac{\partial}{\partial z_{1}} f_{2}\left(z_{1}, z_{2}\right)=0$. Thus, 
the holomorphic functions $f_{1}$ and $f_{2}$ can be rewritten as

$$
\begin{aligned}
& f_{1}\left(z_{1}, z_{2}\right)=-\delta z_{1}+g_{1}\left(z_{2}\right), \\
& f_{2}\left(z_{1}, z_{2}\right)=g_{2}\left(z_{2}\right),
\end{aligned}
$$

where $g_{1}, g_{2}$ are holomorphic functions on a neighborhood of $z_{2}=0$.

Since $M_{P}$ is invariant under $f$, one has

$$
\operatorname{Re} f_{1}\left(i t-P\left(z_{2}\right), z_{2}\right)+P\left(f_{2}\left(i t-P\left(z_{2}\right), z_{2}\right)\right)=0
$$

for all $\left(z_{2}, t\right) \in \Delta_{\epsilon_{0}} \times\left(-\delta_{0}, \delta_{0}\right)$ for some $\epsilon_{0}, \delta_{0}>0$.

It follows from (5) with $t=0$ and (4) that

$$
\delta P\left(z_{2}\right)+\operatorname{Re} g_{1}\left(z_{2}\right)+P\left(g_{2}\left(z_{2}\right)\right)=0
$$

for all $z_{2} \in \Delta_{\epsilon_{0}}$. Since $\nu_{0}(P)=+\infty$, we have $\nu_{0}\left(g_{1}\right)=+\infty$, and hence $g_{1} \equiv 0$. This tells us that

$$
P\left(g_{2}\left(z_{2}\right)\right)=-\delta P\left(z_{2}\right)
$$

for all $z_{2} \in \Delta_{\epsilon_{0}}$. Therefore, Lemmas 1 and 3 tell us that $\left|g^{\prime}(0)\right|=1$ and $\delta=-1$. Hence, $f \in \mathrm{G}_{2}\left(M_{P}, 0\right)$, which finishes the proof.

We note that if $P$ vanishes to infinite order at only the origin, then we have the following corollary.

\section{COROLLARY 1}

Let $\left(M_{P}, 0\right)$ be as in Theorem 1. Assume that

(i) $P\left(z_{2}\right) \not \equiv 0$ on a neighborhood of $z_{2}=0$, and

(ii) $S_{\infty}(P)=\{0\}$.

If $\mathfrak{a} \mathfrak{u t}_{0}\left(M_{P}, 0\right)$ is trivial, then

$$
\operatorname{Aut}\left(M_{P}\right)=\mathrm{G}_{2}\left(M_{P}, 0\right) \oplus \mathrm{T}^{1}\left(M_{P}, 0\right),
$$

where $\mathrm{T}^{1}\left(M_{P}, 0\right)$ denotes the set of all translations $T_{t}^{1}, t \in \mathbb{R}$, defined by $T_{t}^{1}\left(z_{1}, z_{2}\right)=\left(z_{1}+i t, z_{2}\right)$.

\section{Proof}

Let $f \in \operatorname{Aut}\left(M_{P}\right)$ be arbitrary. Since the origin is of infinite type, so is $f(0,0)$. Because of the assumption (ii), we have $P_{\infty}\left(M_{P}\right)=\{(i t, 0): t \in \mathbb{R}\}$. This tells us that $f(0,0)=\left(i t_{0}, 0\right)$ for some $t_{0} \in \mathbb{R}$. Then $T_{-t_{0}}^{1} \circ f \in \operatorname{Aut}\left(M_{P}, 0\right)$. Thus, the proof easily follows from Theorem 1.

In the case that $P$ is positive on a punctured disk $\Delta_{\epsilon_{0}}^{*}, \mathfrak{a u t}_{0}\left(M_{P}, 0\right)$ is at most onedimensional (see [NCM]). Moreover, if $P$ is rotational, that is, $P\left(z_{2}\right) \equiv P\left(\left|z_{2}\right|\right)$, then in [N2] we proved that $\operatorname{Aut}\left(M_{P}, 0\right)=\mathrm{G}_{2}\left(M_{P}, 0\right)=\left\{\left(z_{1}, z_{2}\right) \mapsto\left(z_{1}, e^{i t} z_{2}\right)\right.$ : $t \in \mathbb{R}\}$. Therefore, we only consider the case that $P$ is not rotationally symmetricable, that is, there is no conformal map $\varphi$ with $\varphi(0)=0$ such that $P \circ \varphi\left(z_{2}\right) \equiv$ 
$P \circ \varphi\left(\left|z_{2}\right|\right)$, in which case we showed that $\mathfrak{a u t}_{0}\left(M_{P}, 0\right)=\{0\}$ provided that the connected component of 0 in the zero set of $P$ is $\{0\}$ (see Theorem 3). In addition, this assertion still holds if $P$, defined on a neighborhood $U$ of 0 in $\mathbb{C}$, satisfies the condition (I) (see [N1]), that is,

(I.1) $\lim \sup _{\tilde{U} \ni z \rightarrow 0}\left|\operatorname{Re}\left(b z^{k} \frac{P^{\prime}(z)}{P(z)}\right)\right|=+\infty$,

(I.2) $\limsup _{\tilde{U} \ni z \rightarrow 0}\left|\frac{P^{\prime}(z)}{P(z)}\right|=+\infty$,

for all $k=1,2, \ldots$ and for all $b \in \mathbb{C}^{*}$, where $\tilde{U}:=\{z \in U: P(z) \neq 0\}$. Therefore, as an application of Theorem 1 we obtain the following corollaries.

\section{COROLLARY 2}

Let $\left(M_{P}, 0\right)$ be as in Theorem 1. Assume that

(i) $P$ is not rotationally symmetricable,

(ii) the connected component of 0 in the zero set of $P$ is $\{0\}$, and

(iii) the connected component of 0 in $S_{\infty}(P)$ is $\{0\}$.

Then

$$
\operatorname{Aut}\left(M_{P}, 0\right)=G_{2}\left(M_{P}, 0\right)
$$

COROLLARY 3

Let $\left(M_{P}, 0\right)$ be as in Theorem 1. Assume that

(i) $P\left(z_{2}\right) \not \equiv 0$ on a neighborhood of $z_{2}=0$,

(ii) $P$ satisfies the condition (I), and

(iii) the connected component of 0 in $S_{\infty}(P)$ is $\{0\}$.

Then

$$
\operatorname{Aut}\left(M_{P}, 0\right)=G_{2}\left(M_{P}, 0\right)
$$

\subsection{Proof of Theorem 2}

Proof of Theorem 2

(a) As a consequence of Theorem 5 in Appendix A.2, we see that $\mathfrak{a u t}_{0}\left(M_{P}, 0\right)=0$. Therefore, we shall prove that $\mathfrak{a u t}\left(M_{P}, 0\right)=\mathfrak{g}_{-1} \oplus \mathfrak{g}_{0}$. Indeed, let $H\left(z_{1}, z_{2}\right)=$ $h_{1}\left(z_{1}, z_{2}\right) \partial_{z_{1}}+h_{2}\left(z_{1}, z_{2}\right) \partial_{z_{2}} \in \mathfrak{a u t}\left(M_{P}, 0\right)$ be arbitrary, and let $\left\{\phi_{t}\right\}_{t \in \mathbb{R}} \subset \operatorname{Aut}\left(M_{P}\right)$ be the one-parameter subgroup generated by $H$. Since $\phi_{t}$ is biholomorphic for every $t \in \mathbb{R}$, the set $\left\{\phi_{t}(0): t \in \mathbb{R}\right\}$ is contained in $P_{\infty}\left(M_{P}\right)$. We remark that the connected component of 0 in $P_{\infty}\left(M_{P}\right)$ is $\left\{\left(i t_{1}, i t_{2}\right): t_{1}, t_{2} \in \mathbb{R}\right\}$. Therefore, we have $\phi_{t}(0,0) \subset\left\{\left(i t_{1}, i t_{2}\right): t_{1}, t_{2} \in \mathbb{R}\right\}$. Consequently, we obtain $\operatorname{Re} h_{1}(0,0)=0$ and $\operatorname{Re} h_{2}(0,0)=0$. Hence, the holomorphic vector field $H-i \beta_{1} \partial_{z_{1}}-i \beta_{2} \partial_{z_{2}}$, where $\beta_{j}:=\operatorname{Im} h_{j}(0,0)$ for $j=1,2$, belongs to $\mathfrak{a u t}_{0}\left(M_{P}, 0\right)$, which ends the proof of (a).

(b) By (a), we see that $\mathfrak{a u t}\left(M_{P}, 0\right)=\mathfrak{g}_{-1} \oplus \mathfrak{g}_{0}$, that is, it is generated by $i \partial_{z_{1}}$ and $i \partial_{z_{2}}$. Denote by $\left\{T_{t}^{j}\right\}_{t \in \mathbb{R}}$ the one-parameter subgroups generated by $i \partial_{z_{j}}$ for 
$j=1,2$; that is,

$$
T_{t}^{1}\left(z_{1}, z_{2}\right)=\left(z_{1}+i t, z_{2}\right), \quad T_{t}^{2}\left(z_{1}, z_{2}\right)=\left(z_{1}, z_{2}+i t\right), \quad t \in \mathbb{R} .
$$

For any $f=\left(f_{1}, f_{2}\right) \in \operatorname{Aut}\left(M_{P}, 0\right)$, we define families $\left\{F_{t}^{j}\right\}_{t \in \mathbb{R}}$ of automorphisms by setting $F_{t}^{j}:=f \circ T_{-t}^{j} \circ f^{-1}(j=1,2)$. Then it follows that $\left\{F_{t}^{j}\right\}_{t \in \mathbb{R}}, j=1,2$, are one-parameter subgroups of $\operatorname{Aut}\left(M_{P}\right)$. Since $\mathfrak{a u t}\left(M_{P}, 0\right)=\mathfrak{g}_{-1} \oplus \mathfrak{g}_{0}$, the holomorphic vector fields $H^{j}, j=1,2$, generated by $\left\{F_{t}^{j}\right\}_{t \in \mathbb{R}}, j=1,2$, belong to $\mathfrak{g}_{-1} \oplus \mathfrak{g}_{0}$. This means that there exist real numbers $\delta_{1}^{j}, \delta_{2}^{j}, j=1,2$, such that $H^{j}=i \delta_{1}^{j} \partial_{z_{1}}+i \delta_{2}^{j} \partial_{z_{2}}$ for $j=1,2$, which yield that

$$
F_{t}^{j}\left(z_{1}, z_{2}\right)=\left(z_{1}+i \delta_{1}^{j} t, z_{2}+i \delta_{2}^{j} t\right)=T_{\delta_{1}^{j} t}^{1} \circ T_{\delta_{2}^{j} t}^{2}, \quad j=1,2, t \in \mathbb{R} .
$$

This implies that

$$
f=T_{\delta_{1}^{j} t}^{1} \circ T_{\delta_{2}^{j} t}^{2} \circ f \circ T_{t}^{j}
$$

which is equivalent to

$$
\begin{aligned}
& f_{1}\left(z_{1}, z_{2}\right)=f_{1}\left(z_{1}+i t, z_{2}\right)+i \delta_{1}^{1} t \\
& f_{2}\left(z_{1}, z_{2}\right)=f_{2}\left(z_{1}+i t, z_{2}\right)+i \delta_{2}^{1} t \\
& f_{1}\left(z_{1}, z_{2}\right)=f_{1}\left(z_{1}, z_{2}+i t\right)+i \delta_{1}^{2} t \\
& f_{2}\left(z_{1}, z_{2}\right)=f_{2}\left(z_{1}, z_{2}+i t\right)+i \delta_{2}^{2} t
\end{aligned}
$$

It follows from (6) that

$$
\begin{aligned}
& \frac{\partial}{\partial z_{1}} f_{1}\left(z_{1}, z_{2}\right)=-\delta_{1}^{1}, \\
& \frac{\partial}{\partial z_{1}} f_{2}\left(z_{1}, z_{2}\right)=-\delta_{2}^{1}, \\
& \frac{\partial}{\partial z_{2}} f_{1}\left(z_{1}, z_{2}\right)=-\delta_{1}^{2}, \\
& \frac{\partial}{\partial z_{2}} f_{2}\left(z_{1}, z_{2}\right)=-\delta_{2}^{2},
\end{aligned}
$$

which tells us that

$$
f\left(z_{1}, z_{2}\right)=\left(-\delta_{1}^{1} z_{1}-\delta_{1}^{2} z_{2},-\delta_{2}^{1} z_{1}-\delta_{2}^{2} z_{2}\right)
$$

Since $M_{P}$ is invariant under $f$, one has

$$
\begin{aligned}
\operatorname{Re} & f_{1}\left(i t-P\left(z_{2}\right), z_{2}\right)+P\left(f_{2}\left(i t-P\left(z_{2}\right), z_{2}\right)\right) \\
\quad= & \operatorname{Re}\left(-\delta_{1}^{1}\left(i t-P\left(z_{2}\right)\right)-\delta_{1}^{2} z_{2}\right)+P\left(-\delta_{2}^{1}\left(i t-P\left(z_{2}\right)\right)-\delta_{2}^{2} z_{2}\right) \\
\quad= & \delta_{1}^{1} P\left(z_{2}\right)-\delta_{1}^{2} \operatorname{Re}\left(z_{2}\right)+P\left(\delta_{2}^{1} P\left(z_{2}\right)-\delta_{2}^{2} z_{2}\right)=0
\end{aligned}
$$

for all $\left(z_{2}, t\right) \in \Delta_{\epsilon_{0}} \times\left(-\delta_{0}, \delta_{0}\right)$ for some $\epsilon_{0}, \delta_{0}>0$ small enough.

Since $\nu_{0}(P)=+\infty$, we have $\delta_{1}^{2}=0$. Therefore, putting $z_{2}=t \in\left(-\epsilon_{0}, \epsilon_{0}\right)$ in (7), we obtain

$$
P\left(-\delta_{2}^{2} t+\delta_{2}^{1} P(t)\right)=-\delta_{1}^{1} P(t)
$$


for all $t \in\left(-\epsilon_{0}, \epsilon_{0}\right)$. By the mean value theorem, for each $t \in\left(-\epsilon_{0}, \epsilon_{0}\right)$ there exists a number $\gamma(t) \in[0,1]$ such that

$$
P\left(-\delta_{2}^{2} t+\delta_{2}^{1} P(t)\right)=P\left(-\delta_{2}^{2} t\right)+P^{\prime}\left(-\delta_{2}^{2} t+\gamma(t) \delta_{2}^{1} P(t)\right) \delta_{2}^{1} P(t) .
$$

Because of the fact that the function $P^{\prime}\left(-\delta_{2}^{2} t+\gamma(t) \delta_{2}^{1} P(t)\right)$ vanishes to infinite order at $t=0$, by (8) and (9), one has

$$
P\left(-\delta_{2}^{2} t\right)=\left(-\delta_{1}^{1}+o(1)\right) P(t), \quad t \in\left(-\epsilon_{0}, \epsilon_{0}\right) .
$$

Then it follows from the proof of Lemma 1 that $-\delta_{1}^{1}=-\delta_{2}^{2}=1$.

Now (8) becomes

$$
P\left(t+\delta_{2}^{1} P(t)\right)=P(t)
$$

for all $t \in\left(-\epsilon_{0}, \epsilon_{0}\right)$. By Lemma 2, this equation implies that $\delta_{2}^{1}=0$. Therefore, we conclude that $f=\mathrm{id}$, which finishes the proof of (b).

(c) Denote by $T_{t}^{1}$ and $T_{t}^{2}$ the shifts to imaginary directions of the first and second components

$$
T_{t}^{1}\left(z_{1}, z_{2}\right)=\left(z_{1}+i t, z_{2}\right), \quad T_{t}^{2}\left(z_{1}, z_{2}\right)=\left(z_{1}, z_{2}+i t\right), \quad t \in \mathbb{R} .
$$

Now let $f \in \operatorname{Aut}\left(M_{P}\right)$ be arbitrary. Then $f(0,0)$ is of infinite type. It follows from $S_{\infty}(\tilde{P})=\{0\}$ that we have $P_{\infty}\left(M_{P}\right)=\{(i t, i s): t, s \in \mathbb{R}\}$. Therefore, we get $f(0,0)=\left(i t_{0}, i s_{0}\right)$ for some $t_{0}, s_{0} \in \mathbb{R}$ and we obtain $T_{-t_{0}}^{1} \circ T_{-s_{0}}^{2} \circ f \in \operatorname{Aut}\left(M_{P}, 0\right)=$ \{id\} by (b). The proof of (c) follows.

\section{Analysis of holomorphic tangent vector fields}

In this section, we study the determination of the defining function from holomorphic vector fields. Assume that an infinite-type hypersurface $M_{P}$ is defined by $\rho(z)=\operatorname{Re} z_{1}+P\left(z_{2}\right)$ satisfying conditions (i) and (ii) posed in Theorem 3 . Theorem 3 says that if there are nontrivial holomorphic vector fields vanishing at the origin tangent to $M_{P}$, then the hypersurface $M_{P}$ is rotationally symmetric. The typical example of a rotationally symmetric hypersurface is

$$
M_{P}=\left\{\left(z_{1}, z_{2}\right) \in \mathbf{C}^{2}: \operatorname{Re} z_{1}+\exp \left(-\frac{1}{\left|z_{2}\right|^{\alpha}}\right)=0\right\}
$$

where $\alpha>0$, as in Example 2 in Section 6 .

To prove Theorem 3, we need some lemmas.

\section{LEMMA 7}

Let $P: \Delta_{\epsilon_{0}} \rightarrow \mathbb{R}$ be a $\mathcal{C}^{\infty}$-smooth function satisfying that the connected component of $z=0$ in the zero set of $P$ is $\{0\}$ and that $P$ vanishes to infinite order at $z=0$. If $a, b$ are complex numbers and if $g_{0}, g_{1}, g_{2}$ are $\mathcal{C}^{\infty}$-smooth functions defined on $\Delta_{\epsilon_{0}}$ satisfying

(A1) $g_{0}(z)=O(|z|), g_{1}(z)=O\left(|z|^{\ell}\right)$, and $g_{2}(z)=o\left(|z|^{m}\right)$, and

(A2) $\operatorname{Re}\left[\left(a z^{m}+g_{2}(z)\right) P^{n+1}(z)+b z^{\ell}\left(1+g_{0}(z)\right) P_{z}(z)+g_{1}(z) P(z)\right]=0$ for every $z \in \Delta_{\epsilon_{0}}$ 
for any nonnegative integers $\ell, m$, and $n$ except for the following two cases:

(E1) $\ell=1$ and $\operatorname{Re} b=0$, and

(E2) $m=0$ and $\operatorname{Re} a=0$,

then $a b=0$.

The proof of Lemma 7 for the case that $P$ is positive on $\Delta_{\epsilon_{0}}^{*}$ is given in $[\mathrm{KN}$, Lemma 3] (see also [NCM, Lemma 1]). Furthermore, Lemma 7 follows easily from [KN, Lemma 3] and the following lemma.

\section{LEMMA 8}

Let $P, g_{0}, g_{1}, g_{2}, a, b$ be as in Lemma \%. Suppose that $\gamma:\left[t_{0}, t_{\infty}\right) \rightarrow \Delta_{\epsilon_{0}}^{*}\left(t_{0} \in \mathbb{R}\right)$, where either $t_{\infty} \in \mathbb{R}$ or $t_{\infty}=+\infty$, is a solution of the initial-value problem

$$
\frac{d \gamma(t)}{d t}=b \gamma^{\ell}(t)\left(1+g_{0}(\gamma(t))\right), \quad \gamma\left(t_{0}\right)=z_{0},
$$

where $z_{0} \in \Delta_{\epsilon_{0}}^{*}$ with $P\left(z_{0}\right) \neq 0$, such that $\lim _{t \uparrow t_{\infty}} \gamma(t)=0$. Then $P(\gamma(t)) \neq 0$ for every $t \in\left(t_{0}, t_{\infty}\right)$.

Proof

To obtain a contradiction, we suppose that $P$ has a zero on $\gamma$. Then since the connected component of $z=0$ in the zero set of $P$ is $\{0\}$, without loss of generality we may assume that there exists a $t_{1} \in\left(t_{0}, t_{\infty}\right)$ such that $P(\gamma(t)) \neq 0$ for all $t \in$ $\left(t_{0}, t_{1}\right)$ and $P\left(\gamma\left(t_{1}\right)\right)=0$. Denote $u(t):=\frac{1}{2} \log |P(\gamma(t))|$ for $t_{0}<t<t_{1}$. It follows from (A2) that

$$
u^{\prime}(t)=-P^{n}(\gamma(t))\left(\operatorname{Re}\left(a \gamma^{m}(t)+o\left(|\gamma(t)|^{m}\right)\right)\right)+O\left(|\gamma(t)|^{\ell}\right)
$$

for all $t_{0}<t<t_{1}$. This means that $u^{\prime}(t)$ is bounded on $\left(t_{0}, t_{1}\right)$. Therefore, $u(t)$ is also bounded on $\left(t_{0}, t_{1}\right)$, which contradicts the fact that $u(t) \rightarrow-\infty$ as $t \uparrow t_{1}$. Hence, our lemma is proved.

Following the proof of Lemma 7 (see also [NCM, Lemma 1]), we have the following corollary.

\section{COROLLARY 4}

Let $P: \Delta_{\epsilon_{0}} \rightarrow \mathbb{R}$ be a $\mathcal{C}^{\infty}$-smooth function satisfying that the connected component of $z=0$ in the zero set of $P$ is $\{0\}$ and that $P$ vanishes to infinite order at $z=0$. If $b$ is a complex number and if $g$ is a $\mathcal{C}^{\infty}$-smooth function defined on $\Delta_{\epsilon_{0}}$ satisfying

(B1) $g(z)=O\left(|z|^{k+1}\right)$, and

(B2) $\operatorname{Re}\left[\left(b z^{k}+g(z)\right) P_{z}(z)\right]=0$ for every $z \in \Delta_{\epsilon_{0}}$

for some nonnegative integer $k$, except the case $k=1$ and $\operatorname{Re}(b)=0$, then $b=0$.

Now we are ready to prove Theorem 3. 
Proof of Theorem 3

The CR hypersurface germ $\left(M_{P}, 0\right)$ at the origin in $\mathbb{C}^{2}$ is defined by the equation

$$
\rho\left(z_{1}, z_{2}\right):=\operatorname{Re} z_{1}+P\left(z_{2}\right)=0,
$$

where $P$ is a $\mathcal{C}^{\infty}$-smooth function satisfying the two conditions of this theorem. In particular, recall that $P$ vanishes to infinite order at $z_{2}=0$.

Then we consider a holomorphic vector field $H=h_{1}\left(z_{1}, z_{2}\right) \partial_{z_{1}}+h_{2}\left(z_{1}, z_{2}\right) \partial_{z_{2}}$ defined on a neighborhood of the origin. We only consider $H$ that is tangent to $M_{P}$. This means that they satisfy the identity

$$
(\operatorname{Re} H) \rho(z)=0, \quad \forall z \in M_{P} .
$$

Expand $h_{1}$ and $h_{2}$ into the Taylor series at the origin

$$
\begin{aligned}
& h_{1}\left(z_{1}, z_{2}\right)=\sum_{j, k=0}^{\infty} a_{j k} z_{1}^{j} z_{2}^{k}=\sum_{j=0}^{\infty} a_{j}\left(z_{2}\right) z_{1}^{j}, \\
& h_{2}\left(z_{1}, z_{2}\right)=\sum_{j, k=0}^{\infty} b_{j k} z_{1}^{j} z_{2}^{k}=\sum_{j=0}^{\infty} b_{j}\left(z_{2}\right) z_{1}^{j},
\end{aligned}
$$

where $a_{j k}, b_{j k} \in \mathbb{C}$ and $a_{j}, b_{j}$ are holomorphic functions for every $j \in \mathbb{N}$. We note that $a_{00}=b_{00}=0$ since $h_{1}(0,0)=h_{2}(0,0)=0$.

By a simple computation, we have

$$
\rho_{z_{1}}\left(z_{1}, z_{2}\right)=\frac{1}{2}, \quad \rho_{z_{2}}\left(z_{1}, z_{2}\right)=P_{z_{2}}\left(z_{2}\right)
$$

and (10) can thus be rewritten as

$$
\operatorname{Re}\left[\frac{1}{2} h_{1}\left(z_{1}, z_{2}\right)+P_{z_{2}}\left(z_{2}\right) h_{2}\left(z_{1}, z_{2}\right)\right]=0
$$

for all $\left(z_{1}, z_{2}\right) \in M_{P}$. Since the point $\left(i t-P\left(z_{2}\right), z_{2}\right)$ is in $M_{P}$ with $t$ small enough, the above equation again admits a new form

$$
\operatorname{Re}\left[\frac{1}{2} \sum_{j, k=0}^{\infty} a_{j k}\left(i t-P\left(z_{2}\right)\right)^{j} z_{2}^{k}+P_{z_{2}}\left(z_{2}\right) \sum_{m, n=0}^{\infty} b_{m n}\left(i t-P\left(z_{2}\right)\right)^{m} z_{2}^{n}\right]=0
$$

for all $z_{2} \in \mathbb{C}$ and for all $t \in \mathbb{R}$ with $\left|z_{2}\right|<\epsilon_{0}$ and $|t|<\delta_{0}$, where $\epsilon_{0}>0$ and $\delta_{0}>0$ are small enough. Without loss of generality, we may assume that $H \not \equiv 0$. Since $P_{z_{2}}\left(z_{2}\right)$ vanishes to infinite order at 0 , we notice that if $h_{2} \equiv 0$, then (11) shows that $h_{1} \equiv 0$. So, we must have $h_{2} \not \equiv 0$.

We now divide the argument into two cases as follows.

Case 1: $h_{1} \not \equiv 0$. In this case let us denote by $j_{0}$ the smallest integer such that $a_{j_{0} k} \neq 0$ for some integer $k$. Then let $k_{0}$ be the smallest integer such that $a_{j_{0} k_{0}} \neq 0$. Similarly, let $m_{0}$ be the smallest integer such that $b_{m_{0} n} \neq 0$ for some integer $n$. Then denote by $n_{0}$ the smallest integer such that $b_{m_{0} n_{0}} \neq 0$. We see that $j_{0} \geq 1$ if $k_{0}=0$, and $m_{0} \geq 1$ if $n_{0}=0$. Since $P\left(z_{2}\right)=o\left(\left|z_{2}\right|^{j}\right)$ for any $j \in \mathbb{N}$, 
inserting $t=\alpha P\left(z_{2}\right)$ into (12), where $\alpha \in \mathbb{R}$ will be chosen later, one has

$$
\begin{aligned}
& \operatorname{Re}\left[\frac{1}{2} a_{j_{0} k_{0}}(i \alpha-1)^{j_{0}}\left(P\left(z_{2}\right)\right)^{j_{0}}\left(z_{2}^{k_{0}}+o\left(\left|z_{2}\right|^{k_{0}}\right)\right)\right. \\
& \left.\quad+b_{m_{0} n_{0}}(i \alpha-1)^{m_{0}}\left(z_{2}^{n_{0}}+o\left(\left|z_{2}\right|^{n_{0}}\right)\right)\left(P\left(z_{2}\right)\right)^{m_{0}} P_{z_{2}}\left(z_{2}\right)\right]=0
\end{aligned}
$$

for all $z_{2} \in \Delta_{\epsilon_{0}}$. We note that, in the case $k_{0}=0$ and $\operatorname{Re}\left(a_{j_{0} 0}\right)=0, \alpha$ is chosen in such a way that $\operatorname{Re}\left((i \alpha-1)^{j_{0}} a_{j_{0} 0}\right) \neq 0$. Then (13) yields that $j_{0}>m_{0}$ by virtue of the fact that $P_{z_{2}}\left(z_{2}\right)$ and $P\left(z_{2}\right)$ vanish to infinite order at $z_{2}=0$.

We now consider two subcases as follows.

Subcase 1.1: $m_{0} \geq 1$. If $n_{0}=1$, then the number $\alpha$ can also be chosen such that $\operatorname{Re}\left(b_{m_{0} 1}(i \alpha-1)^{m_{0}}\right) \neq 0$. Therefore, divide $(13)$ by $\left(P\left(z_{2}\right)\right)^{m_{0}}$ to obtain an equation which contradicts Lemma 7 . Hence, we must have $m_{0}=0$.

Subcase 1.2: $m_{0}=0$. In addition to this condition, if $n_{0}>1$, or if $n_{0}=1$ and $\operatorname{Re}\left(b_{01}\right) \neq 0$, then (13) contradicts Lemma 7 . Therefore, we may assume that $n_{0}=1$ and $\operatorname{Re}\left(b_{01}\right)=0$. By a change of variable in $z_{2}$ as in [KN, Lemma 1$]$, we may assume that $b_{0}\left(z_{2}\right) \equiv i z_{2}$.

Next, we shall prove that $b_{m} \equiv 0$ for every $m \in \mathbb{N}^{*}$. Indeed, suppose otherwise. Then let $m_{1}>0$ be the smallest integer such that $b_{m_{1}} \not \equiv 0$. Thus, it can be written as

$$
b_{m_{1}}\left(z_{2}\right)=b_{m_{1} n_{1}} z_{2}^{n_{1}}+o\left(z_{2}^{n_{1}}\right),
$$

where $n_{1}=\nu_{0}\left(b_{m_{1}}\right)$ and $b_{m_{1} n_{1}} \in \mathbb{C}^{*}$. Take a derivative by $t$ at $t=\alpha P\left(z_{2}\right)$ of both sides of (12), and notice that $\nu_{0}(P)=+\infty$. One obtains that

$$
\begin{aligned}
& \operatorname{Re}\left[i m_{1}(\alpha i-1)^{m_{1}-1}\left(P\left(z_{2}\right)\right)^{m_{1}-1}\left(b_{m_{1} n_{1}} z_{2}^{n_{1}}+o\left(\left|z_{2}\right|^{n_{1}}\right)\right) P_{z_{2}}\left(z_{2}\right)\right. \\
& \left.\quad+j_{1}\left(a_{j_{1} k_{1}} z_{2}^{k_{1}}+o\left(\left|z_{2}\right|^{k_{1}}\right)\right)(\alpha i-1)^{j_{1}-1}\left(P\left(z_{2}\right)\right)^{j_{1}-1}\right]=0
\end{aligned}
$$

for all $z_{2} \in \Delta_{\epsilon_{0}}$, where $j_{1}, n_{1} \in \mathbb{N}$ and $a_{j_{1} k_{1}} \in \mathbb{C}$.

Following the argument as above, by Lemma 7 and Corollary 4 , we conclude that $m_{1}=n_{1}=1$ and $b_{1}\left(z_{2}\right) \equiv-\beta_{1} z_{2}\left(1+O\left(z_{2}\right)\right)$ for some $\beta_{1} \in \mathbb{R}^{*}$. We claim that $b_{1}\left(z_{2}\right) \equiv-\beta_{1} z_{2}$. Otherwise, (14) implies that

$$
\operatorname{Re}\left(-i \beta_{1}\left(z_{2}-a z_{2}^{\ell}+o\left(\left|z_{2}\right|^{\ell}\right)\right) P_{z_{2}}\left(z_{2}\right)\right)+O\left(P\left(z_{2}\right)\right) \equiv 0
$$

on $\Delta_{\epsilon_{0}}$ for some $a \in \mathbb{C}^{*}$ and $\ell \geq 2$, which is equivalent to

$$
\operatorname{Re}\left(i z_{2} P_{z_{2}}\left(z_{2}\right)\right) \equiv \operatorname{Re}\left[a z^{\ell}\left(1+O\left(\left|z_{2}\right|\right)\right) P_{z_{2}}\left(z_{2}\right)\right]+O\left(P\left(z_{2}\right)\right)
$$

on $\Delta_{\epsilon_{0}}$ for some $a \in \mathbb{C}^{*}$ and $\ell \geq 2$. On the other hand, since $\nu_{0}(P)=+\infty$, inserting $t=0$ into (12) one has

$$
\operatorname{Re}\left[i z_{2}\left(1-i \beta_{1}\left(1+O\left(\left|z_{2}\right|\right)\right) P\left(z_{2}\right)\right) P_{z_{2}}\left(z_{2}\right)+\left(a_{10}+o(1)\right) P\left(z_{2}\right)\right] \equiv 0
$$

on $\Delta_{\epsilon_{0}}$. Therefore, subtracting (15) from (16) yields

$$
\operatorname{Re}\left[i a z_{2}^{\ell}\left(1+O\left(\left|z_{2}\right|\right)\right) P_{z_{2}}\left(z_{2}\right)+\left(a_{10}+o(1)\right) P\left(z_{2}\right)\right] \equiv 0
$$

on $\Delta_{\epsilon_{0}}$, which is impossible by Lemma 7 . Hence, $b_{1}\left(z_{2}\right) \equiv-\beta_{1} z_{2}$.

Using the same argument as above, we obtain that $b_{m}\left(z_{2}\right)=\beta_{m} i^{m+1} z_{2}$ for every $m \in \mathbb{N}^{*}$, where $\beta_{m} \in \mathbb{R}^{*}$ for every $m \in \mathbb{N}^{*}$. 
Putting $t=\alpha P\left(z_{2}\right)$ in (12), one has

$$
\begin{aligned}
& \operatorname{Re}\left[i z_{2}\left(1+i \beta_{1}(i \alpha-1) P\left(z_{2}\right)+\cdots+i^{m} \beta_{m}(i \alpha-1)^{m} P^{m}\left(z_{2}\right)+\cdots\right) P_{z_{2}}\left(z_{2}\right)\right. \\
& \left.\quad+\left(a_{10}+o(1)\right) P\left(z_{2}\right)\right] \equiv 0
\end{aligned}
$$

on $\Delta_{\epsilon_{0}}$. On the other hand, taking the derivative of both sides of (12) by $t$ at $t=\alpha P\left(z_{2}\right)$, one also has

$$
\begin{aligned}
& \operatorname{Re}\left[i z _ { 2 } \left(i^{2} \beta_{1}+i^{3} 2 \beta_{2}(i \alpha-1) P\left(z_{2}\right)+\cdots\right.\right. \\
& \left.\quad+i^{m+2} m \beta_{m}(i \alpha-1)^{m-1} P^{m}\left(z_{2}\right)+\cdots\right) P_{z_{2}}\left(z_{2}\right) \\
& \left.\quad+\frac{1}{2} \sum_{j=1}^{\infty} \sum_{k=0}^{\infty} j a_{j k}(i \alpha-1)^{j-1} P^{j-1}\left(z_{2}\right) z_{2}^{k}\right] \equiv 0
\end{aligned}
$$

or, equivalently,

$$
\begin{aligned}
& \operatorname{Re}\left[i z _ { 2 } \left(1+i 2 \frac{\beta_{2}}{\beta_{1}}(i \alpha-1) P\left(z_{2}\right)+\cdots\right.\right. \\
& \left.+i^{m} m \frac{\beta_{m}}{\beta_{1}}(\alpha i-1)^{m-1} P^{m}\left(z_{2}\right)+\cdots\right) P_{z_{2}}\left(z_{2}\right) \\
& \left.\quad-\frac{1}{2 \beta_{1}} \sum_{j=1}^{\infty} \sum_{k=0}^{\infty} j a_{j k}(i \alpha-1)^{j-1} P^{j-1}\left(z_{2}\right) z_{2}^{k}\right] \equiv 0
\end{aligned}
$$

on $\Delta_{\epsilon_{0}}$.

Now it follows from (18) and (19) that

$$
2 \beta_{2} / \beta_{1}=\beta_{1}, \quad 3 \beta_{3} / \beta_{1}=\beta_{2}, \quad \ldots, \quad m \beta_{m} / \beta_{1}=\beta_{m-1}, \quad \ldots ;
$$

otherwise, subtracting (18) from (19) one gets an equation depending on $\alpha$ which contradicts Lemma 7 for some $\alpha \in \mathbb{R}$. Therefore, $\beta_{m}=\frac{\left(\beta_{1}\right)^{m}}{m !}$ for all $m \in \mathbb{N}^{*}$ and, hence,

$$
h_{2}\left(z_{1}, z_{2}\right)=i z_{2}\left(1+i \beta_{1} z_{1}+i^{2} \frac{\beta_{1}^{2}}{2 !} z_{1}^{2}+\cdots+i^{m} \frac{\beta_{1}^{m}}{m !} z_{1}^{m}+\cdots\right)=i z_{2} e^{i \beta_{1} z_{1}}
$$

for all $z_{2} \in \Delta_{\epsilon_{0}}$. Moreover, (12) becomes

$$
\operatorname{Re}\left[\frac{1}{2} \sum_{j, k=0}^{\infty} a_{j k}\left(i t-P\left(z_{2}\right)\right)^{j} z_{2}^{k}+i z_{2} P_{z_{2}}\left(z_{2}\right) \exp \left(i \beta_{1}\left(i t-P\left(z_{2}\right)\right)\right)\right]=0
$$

for all $\left(z_{2}, t\right) \in \Delta_{\epsilon_{0}} \times\left(-\delta_{0}, \delta_{0}\right)$.

Denote $f\left(z_{2}, t\right):=\operatorname{Re}\left[\sum_{j, k=0}^{\infty} a_{j k}\left(i t-P\left(z_{2}\right)\right)^{j} z_{2}^{k}\right]$ for $\left(z_{2}, t\right) \in \Delta_{\epsilon_{0}} \times\left(-\delta_{0}, \delta_{0}\right)$. Then (20) tells us that

$$
f\left(z_{2}, t\right)=-2 \operatorname{Re}\left[i z_{2} P_{z_{2}}\left(z_{2}\right) \exp \left(i \beta_{1}\left(i t-P\left(z_{2}\right)\right)\right)\right], \quad \forall\left(z_{2}, t\right) \in \Delta_{\epsilon_{0}} \times\left(-\delta_{0}, \delta_{0}\right) .
$$

This implies that $f\left(z_{2}, t\right)$ vanishes to infinite order at $z_{2}=0$ for every $t$ since $P_{z_{2}}\left(z_{2}\right)$ vanishes to infinite order at $z_{2}=0$ and $f_{t}\left(z_{2}, t\right)=-\beta_{1} f\left(z_{2}, t\right)$. Consequently, one must have $a_{j k}=0$ for every $k \in \mathbb{N}^{*}$ and $j \in \mathbb{N}$ and, thus, 


$$
f\left(z_{2}, t\right)=\operatorname{Re}\left[\sum_{j=0}^{\infty} a_{j 0}\left(i t-P\left(z_{2}\right)\right)^{j}\right] .
$$

Furthermore, the equation $f_{t}\left(z_{2}, 0\right)=-\beta_{1} f\left(z_{2}, 0\right)$ yields

$$
\begin{gathered}
\operatorname{Re}\left(i a_{10}\right)+2 \operatorname{Re}\left(i a_{20}\right)\left(-P\left(z_{2}\right)\right)+o\left(P\left(z_{2}\right)\right) \\
\quad=-\beta_{1}\left(\operatorname{Re}\left(a_{10}\right)\left(-P\left(z_{2}\right)\right)+o\left(P\left(z_{2}\right)\right)\right),
\end{gathered}
$$

which implies that $\operatorname{Re}\left(i a_{10}\right)=0,2 \operatorname{Re}\left(i a_{20}\right)=-\beta_{1} \operatorname{Re}\left(a_{10}\right)=-\beta_{1} a_{10}$. Similarly, it follows from the equation $f_{t t}\left(z_{2}, 0\right)=-\beta_{1} f_{t}\left(z_{2}, 0\right)=\beta_{1}^{2} f\left(z_{2}, 0\right)$ that

$$
\begin{aligned}
& 2 \operatorname{Re}\left(i^{2} a_{20}\right)+3 ! \operatorname{Re}\left(i^{2} a_{30}\right)\left(-P\left(z_{2}\right)\right)+o\left(P\left(z_{2}\right)\right) \\
& =\beta_{1}^{2}\left(\operatorname{Re}\left(a_{10}\right)\left(-P\left(z_{2}\right)\right)+o\left(P\left(z_{2}\right)\right)\right),
\end{aligned}
$$

which again implies that $\operatorname{Re}\left(i^{2} a_{20}\right)=0,3 ! \operatorname{Re}\left(i^{2} a_{30}\right)=\beta_{1}^{2} \operatorname{Re}\left(a_{10}\right)=\beta_{1}^{2} a_{10}$. Continuing this process, we conclude that $a_{m 0}=\frac{\left(i \beta_{1}\right)^{m-1}}{m !} a_{10}$ for every $m \in \mathbb{N}^{*}$ and, hence,

$$
h_{1}\left(z_{1}, z_{2}\right) \equiv a_{10} \frac{e^{i \beta_{1} z_{1}}-1}{i \beta_{1}} .
$$

This implies that $a_{10} \neq 0$ as $h_{1}$ does not vanish identically.

Without loss of generality, we may assume that $a_{10}<0$. The case that $a_{10}>0$ will follow by a similar argument.

Now (20) with $t=0$ is equivalent to

$$
2 \operatorname{Re}\left[i z_{2} P_{z_{2}}\left(z_{2}\right) \exp \left(-i \beta_{1} P\left(z_{2}\right)\right)\right]=a_{10} \frac{\sin \left(\beta_{1} P\left(z_{2}\right)\right)}{\beta_{1}}
$$

for all $z_{2} \in \Delta_{\epsilon_{0}}$. Since $P$ is continuous at $z_{2}=0$, we may assume that $\left|P\left(z_{2}\right)\right|<$ $\frac{\pi}{\left|\beta_{1}\right|}$ for every $\left|z_{2}\right|<\epsilon_{0}$. Moreover, because of the property (i) of $P$ there exists a real number $r \in\left(0, \epsilon_{0}\right)$ such that $0<|P(r)|<\frac{\pi}{\left|\beta_{1}\right|}$ and $r e^{\pi /\left|a_{10}\right|}<\epsilon_{0}$.

Fix $r$, and let $\gamma:(-a, b) \rightarrow \Delta_{\epsilon_{0}}^{*}$, where $a, b \in(0,+\infty)$, be a flow of the equation

$$
\frac{d \gamma(t)}{d t}=i \gamma(t) \exp \left(-i \beta_{1} P(\gamma(t))\right), \quad \gamma(0)=r
$$

Denote $u(t):=P(\gamma(t))$ for $-a<t<b$. Then (21) is equivalent to

$$
u^{\prime}(t)=a_{10} \frac{\sin \left(\beta_{1} u\right)}{\beta_{1}} .
$$

A short computation shows that this differential equation has the solution

$$
P(\gamma(t))=u(t)=\frac{2}{\beta_{1}} \arctan \left\{\tan \left(\beta_{1} P(r) / 2\right) e^{a_{10} t}\right\}, \quad-a<t<b .
$$

Therefore we have, for $-a<t<b$,

$$
\begin{aligned}
\gamma(t) & =r \exp \left[\int_{0}^{t} i e^{-i \beta_{1} P(\gamma(s))} d s\right] \\
& =r \exp \left[\int_{0}^{t} i \exp \left(-2 i \arctan \left\{\tan \left(\beta_{1} P(r) / 2\right) e^{a_{10} s}\right\}\right) d s\right],
\end{aligned}
$$


and thus,

$$
|\gamma(t)|=r \exp \left[\int_{0}^{t} \sin \left(2 \arctan \left\{\tan \left(\beta_{1} P(r) / 2\right) e^{a_{10} s}\right\}\right) d s\right] .
$$

Since $a_{10}<0$, one can choose $a=+\infty$, and by employing some trigonometric identities we obtain

$$
\begin{aligned}
r^{+} & :=\lim _{t \rightarrow-\infty}|\gamma(t)| \\
& =r \exp \left[\int_{0}^{-\infty} \sin \left(2 \arctan \left\{\tan \left(\beta_{1} P(r) / 2\right) e^{a_{10} s}\right\}\right) d s\right] \\
& =r \exp \left[\int_{0}^{-\infty} \sin \left(\pi-2 \arctan \left\{\frac{e^{-a_{10} s}}{\tan \left(\beta_{1} P(r) / 2\right)}\right\}\right) d s\right] \\
& =r \exp \left[\int_{0}^{-\infty} \sin \left(2 \arctan \left\{\frac{e^{-a_{10} s}}{\tan \left(\beta_{1} P(r) / 2\right)}\right\}\right) d s\right] \\
& =r \exp \left[-\int_{0}^{+\infty} \sin \left(2 \arctan \left\{\frac{e^{a_{10} s}}{\tan \left(\beta_{1} P(r) / 2\right)}\right\}\right) d s\right] \\
& =r \exp \left[-2 \int_{0}^{+\infty} \frac{e^{a_{10}}}{1+\left(\frac{e^{a_{10}}}{\tan \left(\beta_{1} P(r) / 2\right)}\right)^{2}} d s\right] \\
& =r \exp \left[-\frac{2}{a_{10}} \int_{0}^{+\infty} \frac{d\left(\frac{e^{a_{10}}}{\tan \left(\beta_{1} P(r) / 2\right)}\right)}{1+\left(\frac{e^{a_{10}}}{\tan \left(\beta_{1} P(r) / 2\right)}\right)^{2}}\right] \\
& =r \exp \left[\frac{2}{a_{10}} \arctan \left(\frac{1}{\tan \left(\beta_{1} P(r) / 2\right)}\right)\right] \\
& \leq r \exp \left(\frac{\pi}{\left|a_{10}\right|}\right)<\epsilon_{0} .
\end{aligned}
$$

Therefore, there exists a sequence $\left\{t_{n}\right\} \subset \mathbb{R}$ such that $t_{n} \rightarrow-\infty$ and $\gamma\left(t_{n}\right) \rightarrow$ $r^{+} e^{i \theta_{0}}$ as $n \rightarrow \infty$ for some $\theta_{0} \in[0,2 \pi)$. Moreover, $\left|P\left(r^{+} e^{i \theta_{0}}\right)\right|<\left|\frac{\pi}{\beta_{1}}\right|$. However, since $a_{10}<0$ and since $P$ is continuous on $\Delta_{\epsilon_{0}}$, it follows from (22) that

$$
\left|P\left(r^{+} e^{i \theta_{0}}\right)\right|=\left|P\left(\lim _{n \rightarrow \infty} \gamma\left(t_{n}\right)\right)\right|=\left|\lim _{n \rightarrow \infty} P\left(\gamma\left(t_{n}\right)\right)\right|=\left|\frac{\pi}{\beta_{1}}\right|,
$$

which is impossible. Therefore, altogether we must have $h_{1} \equiv 0$.

Case 2: $h_{1} \equiv 0$. We shall follow the proof of [N1, Lemma 12]. In this case, (12) is equivalent to

$$
\operatorname{Re}\left[P_{z_{2}}\left(z_{2}\right) \sum_{m=0}^{\infty}\left(i t-P\left(z_{2}\right)\right)^{m} b_{m}\left(z_{2}\right)\right]=0
$$

for all $\left(z_{2}, t\right) \in \Delta_{\epsilon_{0}} \times\left(-\delta_{0}, \delta_{0}\right)$, where $\epsilon_{0}>0$ and $\delta_{0}>0$ are small enough.

Since $h_{2} \not \equiv 0$, there is a smallest $m_{0}$ such that $b_{m_{0}} \not \equiv 0$ and thus it can be written as

$$
b_{m_{0}}\left(z_{2}\right)=b_{m_{0} n_{0}} z_{2}^{n_{0}}+o\left(z_{2}^{n_{0}}\right)
$$


where $n_{0}=\nu_{0}\left(b_{n_{0}}\right)$ and $b_{m_{0} n_{0}} \in \mathbb{C}^{*}$. Moreover, since $P\left(z_{2}\right)=o\left(\left|z_{2}\right|^{n_{0}}\right)$ it follows from (23) with $t=\alpha P\left(z_{2}\right)$ ( $\alpha \in \mathbb{R}$ will be chosen later) that

$$
\operatorname{Re}\left[(i \alpha-1)^{m_{0}}\left(b_{m_{0} n_{0}} z_{2}^{n_{0}}+o\left(\left|z_{2}\right|^{n_{0}}\right)\right) P_{z_{2}}\left(z_{2}\right)\right]=0
$$

for every $z_{2} \in \Delta_{\epsilon_{0}}^{*}$. Notice that if $m_{0}>0$, then we can choose $\alpha$ so that

$$
\operatorname{Re}\left[b_{m_{0} n_{0}}(i \alpha-1)^{m_{0}}\right] \neq 0 .
$$

Therefore, it follows from Corollary 4 that $m_{0}=0, n_{0}=1$, and $\operatorname{Re}\left(b_{m_{0} n_{0}}\right)=$ $\operatorname{Re}\left(b_{01}\right)=0$. By a change of variable in $z_{2}$ (see [N1, Lemma 1$]$ ), we can assume that $b_{0}\left(z_{2}\right) \equiv i z_{2}$.

Next, we shall prove that $b_{m} \equiv 0$ for every $m \in \mathbb{N}^{*}$. Indeed, suppose otherwise. Then by using the same argument as in Subcace $1.1, b_{m}\left(z_{2}\right) \equiv i^{m+1} \frac{\left(\beta_{1}\right)^{m}}{m !} z_{2}$ for every $m \in \mathbb{N}^{*}$. Therefore, $h_{2}\left(z_{2}\right) \equiv i z_{2} e^{i \beta_{1} z_{1}}$.

Now (23) with $t=0$ is equivalent to

$$
2 \operatorname{Re}\left[i z_{2} P_{z_{2}}\left(z_{2}\right) \exp \left(-i \beta_{1} P\left(z_{2}\right)\right)\right]=0
$$

for all $z_{2} \in \Delta_{\epsilon_{0}}$.

Let $\gamma:(-a, b) \rightarrow \Delta_{\epsilon_{0}}^{*}$, where $a, b \in(0,+\infty)$, be a flow of the equation

$$
\frac{d \gamma(t)}{d t}=i \gamma(t) \exp \left(-i \beta_{1} P(\gamma(t))\right), \quad \gamma(0)=r
$$

where $0<r<\epsilon_{0}$ with $P(r) \neq 0$. Denote $u(t):=P(\gamma(t))$ for $-a<t<b$. Then (24) is equivalent to

$$
u^{\prime}(t)=0, \quad-a<t<b .
$$

This tells us that $u(t) \equiv u(0)$, and therefore $P(\gamma(t))=P(r)$ for all $t \in(-a, b)$. Hence, we have

$$
\gamma(t)=r \exp \left(i e^{-i \beta_{1} P(r)} t\right)
$$

for all $t \in(-a, b)$, and thus

$$
|\gamma(t)|=r \exp \left(\sin \left(\beta_{1} P(r)\right) t\right) .
$$

Without loss of generality, we may assume that $\beta_{1} P(r)<0$. Then one can choose $b=+\infty$ and (25) implies that $\gamma(t) \rightarrow 0$ as $t \rightarrow+\infty$. Therefore,

$$
P(r)=P(\gamma(t))=\lim _{t \rightarrow+\infty} P(\gamma(t))=P(0)=0 .
$$

This is a contradiction. Therefore, $h_{2}\left(z_{2}\right) \equiv i z_{2}$.

Consequently, (23) is now equivalent to

$$
\operatorname{Re}\left[i z_{2} P^{\prime}\left(z_{2}\right)\right]=0
$$

for all $z_{2} \in \Delta_{\epsilon_{0}}$, and thus, it follows from [KN, Lemma 4] that $P$ is rotational. This ends the proof. 


\section{Examples}

\section{EXAMPLE 1}

For $\alpha, C>0$, let $P$ be a function given by

$$
P\left(z_{2}\right)= \begin{cases}\exp \left(-\frac{C}{\left|\operatorname{Re}\left(z_{2}\right)\right|^{\alpha}}\right) & \text { if } \operatorname{Re}\left(z_{2}\right) \neq 0, \\ 0 & \text { if } \operatorname{Re}\left(z_{2}\right)=0 .\end{cases}
$$

We note that the function $P$ satisfies condition (I) (see [N1, Example 1]). Moreover, since the function $\tilde{P}$, defined by $\tilde{P}\left(z_{2}\right)=\exp \left(-\frac{C}{\left|z_{2}\right|^{\alpha}}\right)$ if $z_{2} \neq 0$ and $\tilde{P}(0)=0$, vanishes to infinite order only at the origin, it follows from Theorem 2 that $\mathfrak{a u t}_{0}\left(M_{P}, 0\right)=0$ and

$$
\mathfrak{a u t}\left(M_{P}, 0\right)=\mathfrak{g}_{-1} \oplus \mathfrak{g}_{0}=\left\{i \beta_{1} \partial_{z_{1}}+i \beta_{2} \partial_{z_{2}}: \beta_{1}, \beta_{2} \in \mathbb{R}\right\} .
$$

In addition, one obtains that $\operatorname{Aut}\left(M_{P}, 0\right)=\{\operatorname{id}\}$ and $\operatorname{Aut}\left(M_{P}\right)=\left\{\left(z_{1}, z_{2}\right) \mapsto\right.$ $\left.\left(z_{1}+i t, z_{2}+i s\right): t, s \in \mathbb{R}\right\}$.

\section{EXAMPLE 2}

Denote by $M_{P}$ the hypersurface

$$
M_{P}:=\left\{\left(z_{1}, z_{2}\right) \in \mathbb{C}^{2}: \operatorname{Re} z_{1}+P\left(z_{2}\right)=0\right\} .
$$

Let $P_{1}, P_{2}$ be functions given by

$$
\begin{aligned}
& P_{1}\left(z_{2}\right)= \begin{cases}\exp \left(-\frac{1}{\left|z_{2}\right|^{\alpha}}\right) & \text { if } z_{2} \neq 0, \\
0 & \text { if } z_{2}=0,\end{cases} \\
& P_{2}\left(z_{2}\right)= \begin{cases}\exp \left(-\frac{1}{\left|z_{2}\right|^{\alpha}}+\operatorname{Re}\left(z_{2}^{m}\right)\right) & \text { if } z_{2} \neq 0, \\
0 & \text { if } z_{2}=0,\end{cases}
\end{aligned}
$$

where $\alpha>0$ and $m \in \mathbb{N}^{*}$.

It is easy to check that $S_{\infty}\left(P_{1}\right)=S_{\infty}\left(P_{2}\right)=\{0\}$. Moreover, $P_{1}, P_{2}$ are positive on $\mathbb{C}^{*}, P_{1}$ is rotational, and $P_{2}$ is not rotational. Therefore, by Theorems 1,2 and 3, [N2, Theorem B], and Corollaries 1 and 2, we obtain

$$
\begin{aligned}
\mathfrak{a u t}_{0}\left(M_{P_{1}}, 0\right) & =\left\{i \beta z_{2} \partial_{z_{2}}: \beta \in \mathbb{R}\right\}, \\
\mathfrak{a u t}\left(M_{P_{1}}, 0\right) & =\mathfrak{g}_{-1} \oplus \mathfrak{a} \mathfrak{t}_{0}\left(M_{P_{1}}, 0\right) \\
& =\left\{i \beta_{1} \partial_{z_{1}}+i \beta_{2} z_{2} \partial_{z_{2}}: \beta_{1}, \beta_{2} \in \mathbb{R}\right\}, \\
\mathfrak{a u t}_{0}\left(M_{P_{2}}, 0\right) & =0, \\
\mathfrak{a u t}\left(M_{P_{2}}, 0\right) & =\mathfrak{g}_{-1}=\left\{i \beta \partial_{z_{1}}: \beta \in \mathbb{R}\right\}
\end{aligned}
$$

and

$$
\begin{aligned}
\operatorname{Aut}\left(M_{P_{1}}, 0\right) & =\left\{\left(z_{1}, z_{2}\right) \mapsto\left(z_{1}, e^{i t} z_{2}\right): t \in \mathbb{R}\right\}, \\
\operatorname{Aut}\left(M_{P_{1}}\right) & =\operatorname{Aut}\left(M_{P_{1}}, 0\right) \oplus \mathrm{T}^{1}\left(M_{P_{1}}\right) \\
& =\left\{\left(z_{1}, z_{2}\right) \mapsto\left(z_{1}+i s, e^{i t} z_{2}\right): s, t \in \mathbb{R}\right\},
\end{aligned}
$$




$$
\begin{aligned}
\operatorname{Aut}\left(M_{P_{2}}, 0\right) & =\left\{\left(z_{1}, z_{2}\right) \mapsto\left(z_{1}, e^{2 k \pi i / m} z_{2}\right): k=0, \ldots, m-1\right\}, \\
\operatorname{Aut}\left(M_{P_{2}}\right) & =\operatorname{Aut}\left(M_{P_{2}}, 0\right) \oplus \mathrm{T}^{1}\left(M_{P_{2}}\right) \\
& =\left\{\left(z_{1}, z_{2}\right) \mapsto\left(z_{1}+i t, e^{2 k \pi i / m} z_{2}\right): t \in \mathbb{R}, k=0, \ldots, m-1\right\} .
\end{aligned}
$$

\section{Appendix}

\section{A.1 Leau-Fatou flower theorem}

The Leau-Fatou flower theorem states that it is possible to find invariant simple connected domains containing 0 on the boundaries such that, on each domain, a conformal map which is tangent to the identity is conjugated to a parabolic automorphism of the domain and each point in the domain is either attracted to or repelled from 0 . For more details we refer the reader to $[A]$ and $[B]$. These domains are called petals and their existence is predicted by the Leau-Fatou flower theorem. To give a simple statement of such a result, we note that if $g(z)=z+a_{r} z^{r}+O\left(z^{r+1}\right)$ with $r>1$ and $a_{r} \neq 0$, then it is possible to perform a holomorphic change of variables in such a way that $g$ becomes conjugated to $g(z)=z+z^{r}+O\left(z^{r+1}\right)$. The number $r$ is the order of $g$ at 0 . With these preliminary considerations at hand we have the following result.

\section{THEOREM 4 (LEAU-FATOU FLOWER THEOREM)}

Let $g(z)=z+z^{r}+O\left(z^{r+1}\right)$ with $r>1$. Then there exist $2(r-1)$ domains called petals, $P_{j}^{ \pm}$, symmetric with respect to the $(r-1)$ directions $\arg z=2 \pi q /(r-1)$, $q=0, \ldots, r-2$, such that $P_{j}^{+} \cap P_{k}^{+}=\emptyset$ and $P_{j}^{-} \cap P_{k}^{-}=\emptyset$ for $j \neq k, 0 \in \partial P_{j}^{ \pm}$, each petal is biholomorphic to the right half-plane $H$, and $g^{k}(z) \rightarrow 0$ as $k \rightarrow \pm \infty$ for all $z \in P_{j}^{ \pm}$, where $g^{k}=\left(g^{-1}\right)^{-k}$ for $k<0$. Moreover, for all $j$, the map $\left.g\right|_{P_{j}^{ \pm}}$ is holomorphically conjugated to the parabolic automorphism $z \rightarrow z+i$ on $H$.

\section{A.2 Holomorphic tangent vector fields on the tubular model}

In the case that an infinite-type model is tubular, we have the following theorem.

\section{THEOREM 5}

Let $\tilde{P}$ be a $\mathcal{C}^{\infty}$-smooth function defined on a neighborhood of 0 in $\mathbb{C}$ satisfying

(i) $\tilde{P}(x) \not \equiv 0$ on a neighborhood of $x=0$ in $\mathbb{R}$, and

(ii) $\tilde{P}$ vanishes to infinite order at $z_{2}=0$.

Denote by $P$ a $\mathcal{C}^{\infty}$-smooth function defined by setting $P\left(z_{2}\right):=\tilde{P}\left(\operatorname{Re} z_{2}\right)$. Then $\mathfrak{a u t}_{0}\left(M_{P}, 0\right)=0$.

Proof

Suppose that $H=h_{1}\left(z_{1}, z_{2}\right) \partial_{z_{1}}+h_{2}\left(z_{1}, z_{2}\right) \partial_{z_{2}}$ is a holomorphic vector field defined on a neighborhood of the origin satisfying $H(0)=0$. We only consider $H$ that is tangent to $M_{P}$, which means that it satisfies the identity

$$
(\operatorname{Re} H) \rho(z)=0, \quad z \in M_{P} .
$$


Expand $h_{1}$ and $h_{2}$ into the Taylor series at the origin

$$
h_{1}\left(z_{1}, z_{2}\right)=\sum_{j, k=0}^{\infty} a_{j k} z_{1}^{j} z_{2}^{k}, \quad h_{2}\left(z_{1}, z_{2}\right)=\sum_{j, k=0}^{\infty} b_{j k} z_{1}^{j} z_{2}^{k},
$$

where $a_{j k}, b_{j k} \in \mathbb{C}$. We note that $a_{00}=b_{00}=0$ since $h_{1}(0,0)=h_{2}(0,0)=0$.

By a simple computation, we have

$$
\rho_{z_{1}}\left(z_{1}, z_{2}\right)=\frac{1}{2}, \quad \rho_{z_{2}}\left(z_{1}, z_{2}\right)=P_{z_{2}}\left(z_{2}\right)=\frac{1}{2} P^{\prime}(x),
$$

where $x=\operatorname{Re}\left(z_{2}\right)$, and (26) can thus be rewritten as

$$
\operatorname{Re}\left[\frac{1}{2} h_{1}\left(z_{1}, z_{2}\right)+P_{z_{2}}\left(z_{2}\right) h_{2}\left(z_{1}, z_{2}\right)\right]=0
$$

for all $\left(z_{1}, z_{2}\right) \in M_{P}$. Since the point $\left(i t-P\left(z_{2}\right), z_{2}\right)$ is in $M_{P}$ with $t$ small enough, the above equation again admits a new form

$$
\operatorname{Re}\left[\frac{1}{2} \sum_{j, k=0}^{\infty} a_{j k}\left(i t-P\left(z_{2}\right)\right)^{j} z_{2}^{k}+P_{z_{2}}\left(z_{2}\right) \sum_{m, n=0}^{\infty} b_{m n}\left(i t-P\left(z_{2}\right)\right)^{m} z_{2}^{n}\right]=0
$$

for all $z_{2} \in \mathbb{C}$ and for all $t \in \mathbb{R}$ with $\left|z_{2}\right|<\epsilon_{0}$ and $|t|<\delta_{0}$, where $\epsilon_{0}>0$ and $\delta_{0}>0$ are small enough. The goal is to show that $H \equiv 0$. Striving for a contradiction, we suppose that $H \not \equiv 0$. Since $P_{z_{2}}\left(z_{2}\right)$ vanishes to infinite order at 0 , we notice that if $h_{2} \equiv 0$, then (27) shows that $h_{1} \equiv 0$. So, we must have $h_{2} \not \equiv 0$.

We now divide the argument into two cases as follows.

Case 1: $h_{1} \neq \equiv 0$. In this case let us denote by $j_{0}$ the smallest integer such that $a_{j_{0} k} \neq 0$ for some integer $k$. Then let $k_{0}$ be the smallest integer such that $a_{j_{0} k_{0}} \neq 0$. Similarly, let $m_{0}$ be the smallest integer such that $b_{m_{0} n} \neq 0$ for some integer $n$. Then denote by $n_{0}$ the smallest integer such that $b_{m_{0} n_{0}} \neq 0$. We see that $j_{0} \geq 1$ if $k_{0}=0$, and $m_{0} \geq 1$ if $n_{0}=0$. Since $P\left(z_{2}\right)=o\left(\left|z_{2}\right|^{j}\right)$ for any $j \in \mathbb{N}$, inserting $t=\alpha P\left(z_{2}\right)$ into (28), where $\alpha \in \mathbb{R}$ will be chosen later, one has

$$
\begin{aligned}
\operatorname{Re} & {\left[\frac{1}{2} a_{j_{0} k_{0}}(i \alpha-1)^{j_{0}}\left(P\left(z_{2}\right)\right)^{j_{0}}\left(z_{2}^{k_{0}}+o\left(\left|z_{2}\right|^{k_{0}}\right)\right)\right.} \\
& \left.+b_{m_{0} n_{0}}(i \alpha-1)^{m_{0}}\left(z_{2}^{n_{0}}+o\left(\left|z_{2}\right|^{n_{0}}\right)\right)\left(P\left(z_{2}\right)\right)^{m_{0}} P_{z_{2}}\left(z_{2}\right)\right]=0
\end{aligned}
$$

for all $z_{2} \in \Delta_{\epsilon_{0}}$. We note that, in the case $k_{0}=0$ and $\operatorname{Re}\left(a_{j_{0} 0}\right)=0, \alpha$ is chosen in such a way that $\operatorname{Re}\left((i \alpha-1)^{j_{0}} a_{j_{0} 0}\right) \neq 0$. Then (29) yields that $j_{0}>m_{0}$ by virtue of the fact that $P_{z_{2}}\left(z_{2}\right)$ and $P\left(z_{2}\right)$ vanish to infinite order at $z_{2}=0$. Moreover, we remark that $P_{z_{2}}\left(z_{2}\right)=\frac{1}{2} P^{\prime}(x)$, where $x:=\operatorname{Re}\left(z_{2}\right)$. Therefore, it follows from (29) that

$$
\frac{P^{\prime}(x)}{(P(x))^{j_{0}-m_{0}}}=\frac{\operatorname{Re}\left[a_{j_{0} k_{0}}(i \alpha-1)^{j_{0}}\left(z_{2}^{k_{0}}+o\left(\left|z_{2}\right|^{k_{0}}\right)\right)\right]}{\operatorname{Re}\left[b_{m_{0} n_{0}}(i \alpha-1)^{m_{0}}\left(z_{2}^{n_{0}}+o\left(\left|z_{2}\right|^{n_{0}}\right)\right)\right]}
$$

for all $z_{2}=x+i y \in \Delta_{\epsilon_{0}}$ satisfying

$$
P(x) \neq 0, \quad \operatorname{Re}\left[b_{m_{0} n_{0}}(i \alpha-1)^{m_{0}}\left(z_{2}^{n_{0}}+o\left(\left|z_{2}\right|^{n_{0}}\right)\right)\right] \neq 0 .
$$

However, (30) is a contradiction since its right-hand side depends also on $y$ and, hence, one must have $h_{1} \equiv 0$. 
Case 2: $h_{1} \equiv 0$. Let $m_{0}, n_{0}$ be as in Case 1. Since $P\left(z_{2}\right)=o\left(\left|z_{2}\right|^{n_{0}}\right)$, putting $t=\alpha P\left(z_{2}\right)$ in (28), where $\alpha \in \mathbb{R}$ will be chosen later, one obtains that

$$
\frac{1}{2} P^{\prime}(x) \operatorname{Re}\left[(i \alpha-1)^{m_{0}} b_{m_{0} n_{0}}\left(z_{2}^{n_{0}}+o\left(\left|z_{2}\right|^{n_{0}}\right)\right)\right]=0
$$

for all $z_{2}=x+i y \in \Delta_{\epsilon_{0}}$. Since $P^{\prime}(x) \not \equiv 0$, one has

$$
\operatorname{Re}\left[(i \alpha-1)^{m_{0}} b_{m_{0} n_{0}}\left(z_{2}^{n_{0}}+o\left(\left|z_{2}\right|^{n_{0}}\right)\right)\right]=0
$$

for all $z_{2} \in \Delta_{\epsilon_{0}}$. Note that if $n_{0}=0$, then $\alpha$ can be chosen in such a way that $\operatorname{Re}\left((i \alpha-1)^{m_{0}} b_{m_{0} 0}\right) \neq 0$. Hence, (31) is absurd.

Altogether, the proof of our theorem is complete.

\section{References}

[A] M. Abate, "Discrete holomorphic local dynamical systems" in Holomorphic Dynamical Systems, Lecture Notes in Math. 1998, Springer, Berlin, 2010, 1-55. MR 2648687. DOI 10.1007/978-3-642-13171-4_1.

[B] F. Bracci, Local dynamics of holomorphic diffeomorphisms, Boll. Unione Mat. Ital. 7 (2004), 609-636. MR 2101654.

[CM] S. S. Chern and J. K. Moser, Real hypersurfaces in complex manifolds, Acta Math. 133 (1974), 219-271. MR 0425155.

[D] J. P. D'Angelo, Real hypersurfaces, orders of contact, and applications, Ann. of Math. (2) 115 (1982), 615-637. MR 0657241. DOI 10.2307/2007015.

[EKS1] V. Ezhov, M. Kolář, and G. Schmalz, Degenerate hypersurfaces with a two-parametric family of automorphisms, Complex Var. Elliptic Equ. 54 (2009), 283-291. MR 2513540. DOI 10.1080/17476930902760443.

[EKS2] _ Normal forms and symmetries of real hypersurfaces of finite type in $\mathbb{C}^{2}$, Indiana Univ. Math. J. 62 (2013), 1-32. MR 3158500.

DOI 10.1512/iumj.2013.62.4833.

[KN] K.-T. Kim and N. V. Thu, On the tangential holomorphic vector fields vanishing at an infinite type point, Trans. Amer. Math. Soc. 367 (2015), 867-885. MR 3280030. DOI 10.1090/S0002-9947-2014-05917-5.

[K1] M. Kolář, Normal forms for hypersurfaces of finite type in $\mathbb{C}^{2}$, Math. Res. Lett. 12 (2005), 897-910. MR 2189248. DOI 10.4310/MRL.2005.v12.n6.a10.

[K2] Local symmetries of finite type hypersurfaces in $\mathbb{C}^{2}$, Sci. China Ser. A 49 (2006), 1633-1641. MR 2288220. DOI 10.1007/s11425-006-2049-6.

[K3] Local equivalence of symmetric hypersurfaces in $\mathbb{C}^{2}$, Trans. Amer. Math. Soc. 362, no. 6 (2010), 2833-2843. MR 2592937.

DOI 10.1090/S0002-9947-10-05058-0.

[KM] M. Kolář and F. Meylan, Infinitesimal CR automorphisms of hypersurfaces of finite type in $\mathbb{C}^{2}$, Arch. Math. (Brno) 47 (2011), 367-375. MR 2876940.

[KMZ] M. Kolář, F. Meylan, and D. Zaitsev, Chern-Moser operators and polynomial models in CR geometry, Adv. Math. 263 (2014), 321-356.

MR 3239141. DOI 10.1016/j.aim.2014.06.017. 
[N1] V. T. Ninh, On the existence of tangential holomorphic vector fields vanishing at an infinite type point, preprint, arXiv:1303.6156v7 [math.CV].

[N2] - On the CR automorphism group of a certain hypersurface of infinite type in $\mathbb{C}^{2}$, Complex Var. Elliptic Equ. 60 (2015), 977-991. DOI 10.1080/17476933.2014.986656.

[NCM] V. T. Ninh, V. T. Chu, and A. D. Mai, On the real-analytic infinitesimal $C R$ automorphism of hypersurfaces of infinite type, preprint, arXiv:1404.4914v2 [math.CV].

[S1] N. K. Stanton, Infinitesimal CR automorphisms of rigid hypersurfaces, Amer. J. Math. 117 (1995), 141-167. MR 1314461. DOI 10.2307/2375039. Infinitesimal CR automorphisms of real hypersurfaces, Amer. J. Math. 118 (1996), 209-233. MR 1375306.

Hayashimoto: Nagano National College of Technology, Nagano, Japan; atsushi@nagano-nct.ac.jp

Thu: Center for Geometry and Its Applications, Pohang University of Science and Technology, Pohang, Republic of Korea; current address: Department of Mathematics, Vietnam National University at Hanoi, Hanoi, Vietnam; thunv@vnu.edu.vn 